\title{
Financial Market Models with Lévy Processes and Time-Varying Volatility
}

Young Shin Kim, Department of Econometrics, Statistics and Mathematical Finance School of Economics and Business Engineering. University of Karlsruhe

Svetlozar T. Rachev, Chair-Professor, Chair of Econometrics, Statistics and Mathematical Finance, School of Economics and Business Engineering University of Karlsruhe and Department of Statistics and Applied Probability, University of California, Santa Barbara Michele Leonardo Bianchi, Department of Mathematics, Statistics, Computer Science and Applications, University of Bergamo

Frank J. Fabozzi, School of Management, Yale University

Acknowledgments: Rachev's research was supported by grants from the Division of Mathematical, Life and Physical Science, College of Letters and Science, University of California, Santa Barbara, and the Deutschen Forschungsgemeinschaft. Bianchi is grateful for research support provided by the German Academic Exchange Service (DAAD).

\author{
Contact Author: Svetlozar T.Rachev \\ Chair of Econometrics, Statistics and Mathematical Finance \\ School of Economics and Business Engineering \\ University of Karlsruhe \\ Kollegium am Schloss, Bau II, 20.12, R210 \\ Postfach 6980, D-76128, Karlsruhe, Germany \\ Tel: +49-721-608-7535, 0+49-721-608-2042(s) \\ FAX: +49-721-608-3811 \\ E-mail: rachev@statistik.uni-karlsruhe.de \\ http://www.statistik.uni-karlsruhe.de
}




\title{
Financial Market Models with Lévy Processes and Time-Varying Volatility
}

\begin{abstract}
Asset management and pricing models require the proper modeling of the return distribution of financial assets. While the return distribution used in the traditional theories of asset pricing and portfolio selection is the normal distribution, numerous studies that have investigated the empirical behavior of asset returns in financial markets throughout the world reject the hypothesis that asset return distributions are normally distribution. Alternative models for describing return distributions have been proposed since the 1960s, with the strongest empirical and theoretical support being provided for the family of stable distributions (with the normal distribution being a special case of this distribution). Since the turn of the century, specific forms of the stable distribution have been proposed and tested that better fit the observed behavior of historical return distributions. More specifically, subclasses of the tempered stable distribution have been proposed. In this paper, we propose one such subclass of the tempered stable distribution which we refer to as the "KR distribution". We empirically test this distribution as well as two other recently proposed subclasses of the tempered stable distribution: the Carr-Geman-Madan-Yor (CGMY) distribution and the modified tempered stable (MTS) distribution. The advantage of the KR distribution over the other two distributions is that it has more flexible tail parameters. For these three subclasses of the tempered stable distribution, which are infinitely divisible and have exponential moments for some neighborhood of zero, we generate the exponential Lévy market models induced from them. We then construct a new GARCH model with the infinitely divisible distributed innovation and three subclasses of that GARCH model that incorporates three observed properties of asset returns: volatility clustering, fat tails, and skewness. We formulate the algorithm to find the risk-neutral return processes for those GARCH models using the "change of measure" for the tempered stable distributions. To compare the performance of those exponential Lévy models and the GARCH models, we report the results of the parameters estimated for the S\&P 500 index and investigate the out-of-sample forecasting performance for those GARCH models for the S\&P 500 option prices.
\end{abstract}

Keywords: Tempered stable distribution, GARCH model, Option pricing.

JEL Classifications: G12, G20 


\section{Introduction}

Since Mandelbrot (1963) introduced the Lévy stable (or $\alpha$-stable) distribution to model the empirical distribution of asset prices, the $\alpha$-stable distribution became the most popular alternative to the normal distribution, the latter distribution being rejected by numerous empirical studies that have found financial return series to be heavy-tailed and possibly skewed. Rachev and Mittnik (2000) and Rachev et al. (2005) have developed financial models with $\alpha$-stable distributions and applied them to market and credit risk management, option pricing, and portfolio selection as will as discussing the major attacks on the $\alpha$-stable models. A fair conclusion of the literature is that while the empirical evidence does not support the normal distribution, it is also not consistent with an $\alpha$-stable distribution. The distribution of returns for assets has heavier tails relative to the normal distribution and thinner tails than the $\alpha$-stable distribution. Partly in response to those empirical inconsistencies, various alternatives to the $\alpha$-stable distribution were proposed in the literature. The "classical tempered stable" (CTS) distribution (Koponen (1995), Boyarchenko and Levendorskii (2000), and Carr et al. (2002)) and the "modified tempered stable" (MTS) distribution (Kim, Rachev, and Chung (2006)) are two examples; an extension of the CTS distribution named the "KR" distribution (Kim et al. (2007)) is another. These distributions, sometimes called the tempered stable distributions, have not only heavier tails than the normal distribution and thinner than the $\alpha$-stable distribution, but also have finite moments for all orders.

The tempered stable distributions are used for constructing the exponential Lévy model. If the driving process is the CTS process, then the exponential Lévy model is called the CGMY model, and if the driving process is the MTS process or KR process, then we refer to the exponential Lévy models as the MTS model or the KR model, respectively.

The main problem with the exponential Lévy models is that they generate an incomplete market; that is, the equivalent martingale measure (EMM) of a given market measure is not unique in general. For this reason, we need a method to select one reasonable EMM in the incomplete market generated by an exponential Lévy model. One classical method in selecting an EMM is the Esscher transform presented by Gerber and Shiu (1994, 1996); another reasonable method is finding the "minimal entropy martingale measure" presented by Fujiwara and Miyahara (2003). While these methods are mathematically elegant and have 
a financial interpretation within the context of a utility maximization problem, empirically the model prices obtained from the EMM have not matched the market prices observed for options. The other method for handling the problem is to estimate the risk-neutral measure by using current option price data independent of the historical underlying distribution. This method can fit model prices to market prices directly, but it has a problem: the historical market measure and the risk-neutral measure need not to be equivalent and it conflicts with the the no-arbitrage property for option prices. To overcome these drawbacks, one must estimate the market measure and the risk-neutral measure simultaneously, and preserve the equivalent property between two measures. One method for doing so is "the least-squares calibration with a prior" proffered by Cont and Tankov (2004). Basically, their method finds an EMM of the market measure that minimizes the least squares error of the model option prices relative to the market option prices.

In spite of the skewness and the fat-tail property of the driving process, the exponential Lévy model has been rejected by empirical evidence (e.g, the finding that there is volatility clustering). The Markov property of the exponential Lévy model is one reason for the rejection. GARCH option pricing models have been developed to price options under the assumption of a non-Markovian property, more precisely, the assumption of volatility clustering. GARCH models of Duan (1995) and Heston and Nandi (2000) are important works on the non-Markovian structure of asset returns with the normal innovation process, but the normal innovation process disregards the empirical innovation process of asset returns. Duan et al. (2004) enhanced the classical GARCH model by adding jumps to the innovation processes. Subsequently, Menn and Rachev (2005a,b) introduced an enhanced GARCH model with innovations which follow the smoothly truncated stable (STS) distribution.

In this paper, we present market models based on the tempered stable distributions and provide empirical tests of these distributions. First, we consider the the CGMY, MTS, and KR models. Then we find their EMM using the method of least-squares calibration with a prior and verify empirically the advantages of the KR model. We can find the parameters of the EMM such that the least squares error of the KR model prices are less than the error of the CGMY and MTS model prices. The change of measure between two KR processes has more freedom than that of the CGMY and MTS, and this freedom provides some empirical 
benefit which will be discussed.

We then construct a new GARCH model that combines the volatility clustering property of Daun's GARCH model and the skewness and fat-tail property of the infinitely divisible distribution which induces the Lévy process. This combination approach was first attempted by Menn and Rachev (2005a,b) using the $\alpha$-stable distribution. In this paper, we improve and extend their approach. We consider the GARCH model and apply infinitely divisible distribution for modeling the residual distribution of the GARCH model. Technically, we apply the three tempered stable distributions (the CTS, the MTS, and the KR distributions) to modeling the residual distribution. The market parameters of the GARCH models are estimated for S\&P 500 index and the prices of three individual stocks. The out-of-sample forecasting performance of those GARCH models for the S\&P 500 call option prices is investigated and compared to the performance of the Black-Scholes model with the historical volatility, the CGMY model with Esscher transform, and the Duan's normal-GARCH model. The implied volatility curves of the call option prices computed using the three tempered stable GARCH option pricing models are also compared to the curves calculated using the market prices and the model prices of the CGMY model and the normal-GARCH model.

The contribution of this paper is threefold. First, we present a new market model which is an extension of the CGMY model that allows for flexible modeling of the tail behavior of the return distribution. We verify the theoretical and empirical benefit of the market model generated by the distribution. Second, we construct new GARCH models with non-normally distributed innovation with flexible tail characteristics. Finally, we formulate a method to ascertain the risk-neutral stock price process corresponding to the new GARCH model and obtain good empirical performance when applying this model to option prices.

The remainder of this paper is organized as follows. Section 2 presents the continuous-time market model and reviews the CGMY model. The MTS distribution and the MTS model are given in Section 3, while the KR distribution and the KR model are given in Section 4. The empirical tests are provided in Section 5 where we show the estimation results for the market parameters and test the calibration performance of the risk-neutral parameters for the CGMY, MTS, and KR models. The GARCH model with the innovations with the infinitely divisible law and its tempered stable subclasses are discussed in Section 6, and its 
empirical results are reported in Section 7. Section 8 summarizes the principal conclusions of the paper.

\section{Continuous-Time Market and CGMY Model}

Let us denote a time horizon by $T>0$ and the risk free rate by $r>0$. Let $\Omega$ be the set of all cadlag functions on $[0, T]$ into $\mathbb{R}$, and $\left(X_{t}\right)_{t \in[0, T]}$ is a canonical process on $\Omega$ (i.e., $\left.X_{t}(\omega)=\omega(t), t \in[0, T], \omega \in \Omega\right)$. Consider the filtered probability space $\left(\Omega, \mathcal{F}_{T},\left(\mathcal{F}_{t}\right)_{t \in[0, T]}\right)$ where $\mathcal{F}_{T}=\sigma\left\{X_{s} ; s \in[0, T]\right\}$ and $\mathcal{F}_{t}=\cap_{s \in(t, T]} \sigma\left\{X_{u}: u \leq s\right\}, t \in[0, T] .\left(\mathcal{F}_{t}\right)_{t \in[0, T]}$ is the right continuous natural filtration. The continuous-time market is modeled by a probability space $\left(\Omega, \mathcal{F}_{T},\left(\mathcal{F}_{t}\right)_{t \in[0, T]}, \mathbb{P}\right)$, for some measure $\mathbb{P}$ named the market measure. In the market, the stock price is given by the random variable $S_{t}=S_{0} e^{X_{t}}, t \in[0, T]$ for some initial value of the stock price $S_{0}>0$. The process $\left(S_{t}\right)_{t \in[0, T]}$ is called the stock price process. The process $\left(X_{t}\right)_{t \in[0, T]}$ is called the driving process of $\left(S_{t}\right)_{t \in[0, T]}$. Assume a stock buyer receives a continuous dividend yields $d$. A probability measure $\mathbb{Q}$ equivalent to $\mathbb{P}$ is called an equivalent martingale measure $(\mathrm{EMM})$ of $\mathbb{P}$ if the process $\left(\hat{S}_{t}\right)_{t \in[0, T]}$ is a $\mathbb{Q}$-martingale where $\hat{S}_{t}=e^{-(r-d) t} S_{t}$.

The classical tempered stable process has been introduced under different names including: "truncated Lévy flight" (Koponen (1995)), "KoBoL" process (Boyarchenko and Levendorskii (2000)), and "CGMY" process (Carr et al. (2002)).

Definition 2.1. An infinitely divisible random variable $X$ is said to follow the classical tempered stable (CTS) distribution if the characteristic function of $X$ is given by

$$
\begin{aligned}
\phi_{X}\left(u ; \alpha, C_{1}, C_{2}, \lambda_{+}, \lambda_{-}, m\right)=\exp (i u m & +C_{1} \Gamma(-\alpha)\left(\left(\lambda_{+}-i u\right)^{\alpha}-\lambda_{+}{ }^{\alpha}\right) \\
& \left.\left.+C_{2} \Gamma(-\alpha)\left(\left(\lambda_{-}+i u\right)^{\alpha}-\lambda_{-}{ }^{\alpha}\right)\right)\right),
\end{aligned}
$$

where $C_{1}, C_{2}, \lambda_{+}, \lambda_{-}>0, \alpha \in(0,2)$ and $m \in \mathbb{R}$, and we denote $X \sim \operatorname{CTS}\left(\alpha, C_{1}, C_{2}, \lambda_{+}, \lambda_{-}\right.$, $m)$. A Lévy process induced from the CTS distribution is called a classical tempered stable (CTS) process with parameters $\left(\alpha, C_{1}, C_{2}, \lambda_{+}, \lambda_{-}, m\right)$.

Remark 2.2. The distribution defined in Definition 2.1 has been called the "tempered stable" or the KoBoL distribution, but Rosinski (2006) extended the distribution under the name of the tempered stable distribution. For that reason, we rename the former as the classical 
tempered stable distribution. The truncated Lévy flight is obtained if $\lambda_{+}=\lambda_{-}$, while the $C G M Y$ distribution is obtained if $C_{1}=C_{2}$.

Using the characteristic function, we can get cumulants $c_{n}(X):=\left.\frac{1}{i^{n}} \frac{d^{n}}{d u^{n}} \log E\left[e^{i u X}\right]\right|_{u=0}$ of the CTS distributed random variable $X$ that

$$
\begin{aligned}
& c_{1}(X)=m+\Gamma(1-\alpha)\left(C_{1}{\lambda_{+}}^{\alpha-1}-C_{2} \lambda_{-}{ }^{\alpha-1}\right), \quad \text { for } \quad n=1 \\
& c_{n}(X)=C_{1} \Gamma(n-\alpha) \lambda_{1}^{\alpha-n}+(-1)^{n} C_{2} \Gamma(n-\alpha) \lambda_{2}^{\alpha-n}, \quad \text { for } \quad n=2,3, \cdots .
\end{aligned}
$$

If we put

$$
\begin{aligned}
& C=C_{1}=C_{2}=\left(\Gamma(2-\alpha)\left(\lambda_{+}{ }^{\alpha-2}+\lambda_{-}{ }^{\alpha-2}\right)\right)^{-1} \\
& m=-\Gamma(1-\alpha)\left(C_{1} \lambda_{+}{ }^{\alpha-1}-C_{2} \lambda_{-}{ }^{\alpha-1}\right),
\end{aligned}
$$

then $X \sim \operatorname{CTS}\left(\alpha, C_{1}, C_{2}, \lambda_{+}, \lambda_{-}, m\right)$ has zero mean and unit variance, and $X$ will be called the standard CTS distribution and denoted by $X \sim \operatorname{stdCTS}\left(\alpha, \lambda_{+}, \lambda_{-}\right)$. Moreover, the log-Laplace transform of $X$ will be denoted by $L_{C T S}\left(x ; \alpha, \lambda_{+}, \lambda_{-}\right)$. The function $L_{C T S}\left(x ; \alpha, \lambda_{+}, \lambda_{-}\right)$is defined on $x \in\left(-\lambda_{-}, \lambda_{+}\right)$and we can obtain

$$
\begin{aligned}
& L_{C T S}\left(x ; \alpha, \lambda_{+}, \lambda_{-}\right) \\
& =\frac{\left(\lambda_{+}-x\right)^{\alpha}-\lambda_{+}{ }^{\alpha}+\left(\lambda_{-}+x\right)^{\alpha}-\lambda_{-}{ }^{\alpha}}{\alpha(\alpha-1)\left({\lambda_{+}}^{\alpha-2}+\lambda_{-}{ }^{\alpha-2}\right)}-\frac{x\left(\lambda_{+}{ }^{\alpha-1}-\lambda_{-}{ }^{\alpha-1}\right)}{(1-\alpha)\left({\lambda_{+}}^{\alpha-2}+\lambda_{-}{ }^{\alpha-2}\right)}
\end{aligned}
$$

by the characteristic function (1).

Now, we make use of the following proposition proven by Kim and Lee (2007) to find an equivalent measure for CTS processes.

Proposition 2.3. Suppose $\left(X_{t}\right)_{t \in[0, T]}$ is the CTS process with parameters $\left(\alpha, C_{1}, C_{2}, \lambda_{+}\right.$, $\left.\lambda_{-}, m\right)$ under $\mathbb{P}$ and the CTS process with parameters $\left(\tilde{\alpha}, \tilde{C}_{1}, \tilde{C}_{2}, \tilde{\lambda}_{+}, \tilde{\lambda}_{-}, \tilde{m}\right)$ under $\mathbb{Q}$. Then $\left.\mathbb{P}\right|_{\mathcal{F}_{t}}$ and $\left.\mathbb{Q}\right|_{\mathcal{F}_{t}}$ are equivalent for all $t>0$ if and only if $\alpha=\tilde{\alpha}, C_{1}=\tilde{C}_{1}, C_{2}=\tilde{C}_{2}$, and $m=\tilde{m}$.

Applying Proposition 2.3 to CTS distributed random variables, we obtain the following corollary.

Corollary 2.4. (a) Let $X \sim \operatorname{CTS}\left(\alpha, C_{1}, C_{2}, \lambda_{+}, \lambda_{-}, m\right)$ under a measure $\mathbf{P}$, and $X \sim$ $\operatorname{CTS}\left(\tilde{\alpha}, \tilde{C}_{1}, \tilde{C}_{2}, \tilde{\lambda}_{+}, \tilde{\lambda}_{-}, \tilde{m}\right)$ under a measure $\mathbf{Q}$. Then $\mathbf{P}$ and $\mathbf{Q}$ are equivalent if and only 
if $\alpha=\tilde{\alpha}, C_{1}=\tilde{C}_{1}, C_{2}=\tilde{C}_{2}$, and $m=\tilde{m}$.

(b) Let $X \sim \operatorname{stdCTS}\left(\alpha, \lambda_{+}, \lambda_{-}\right)$under a measure $\mathbf{P}$, and $(X+k) \sim \operatorname{stdCTS}\left(\tilde{\alpha}, \tilde{\lambda}_{+}, \tilde{\lambda}_{-}\right)$ under a measure $\mathbf{Q}$ for a constant $k \in \mathbb{R}$. Then $\mathbf{P}$ and $\mathbf{Q}$ are equivalent if and only if

$$
\left(\begin{array}{l}
\alpha=\tilde{\alpha}, \\
\tilde{\lambda}_{+}^{\alpha-2}+\tilde{\lambda}_{-}^{\alpha-2}=\lambda_{+}{ }^{\alpha-2}+\lambda_{-}{ }^{\alpha-2} \\
k=\frac{\lambda_{+}{ }^{\alpha-1}-\lambda_{-}{ }^{\alpha-1}-\tilde{\lambda}_{+}^{\alpha-1}+\tilde{\lambda}_{-}^{\alpha-1}}{(1-\alpha)\left(\lambda_{+}{ }^{\alpha-2}+\lambda_{-}{ }^{\alpha-2}\right)} .
\end{array}\right.
$$

The CGMY model is defined under the continuous-time market as follows. For convenience, let us denote

$$
\left.\psi_{C G M Y}^{0}\left(u ; \alpha, C, \lambda_{+}, \lambda_{-}\right):=C \Gamma(-\alpha)\left(\left(\lambda_{+}-i u\right)^{\alpha}-{\lambda_{+}}^{\alpha}+\left(\lambda_{-}+i u\right)^{\alpha}-\lambda_{-}{ }^{\alpha}\right)\right) .
$$

Definition 2.5. Let $C>0, \lambda_{-}>0, \lambda_{+}>1, \alpha \in(0,2)$, and $\mu>0$. In the continuous-time market, if the driving process $\left(X_{t}\right)_{t \in[0, T]}$ of $\left(S_{t}\right)_{t \in[0, T]}$ is a CTS process with parameters $(\alpha$, $\left.C, C, \lambda_{+}, \lambda_{-}, m\right)$ and $m=\mu-\psi_{C G M Y}^{0}\left(-i ; \alpha, C, \lambda_{+}, \lambda_{-}\right)$, then $\left(S_{t}\right)_{t \in[0, T]}$ is called the CGMY stock price process with parameters $\left(\alpha, C, \lambda_{+}, \lambda_{-}, \mu\right)$ and we say that the stock price process follows the CGMY model.

The function $\psi_{C G M Y}^{0}\left(-i ; \alpha, C, \lambda_{+}, \lambda_{-}\right)$is well defined with the condition $\lambda_{+}>1$, and hence $E\left[S_{t}\right]=S_{0} e^{\mu t}, t \in[0, T]$. If we apply Proposition 2.3 to the CGMY model, we obtain the following proposition.

Theorem 2.6 (EMM conditions for the CGMY model). Assume that $\left(S_{t}\right)_{t \in[0, T]}$ is the CGMY stock price process with parameters $\left(\alpha, C, \lambda_{+}, \lambda_{-}, \mu\right)$ under the market measure $\mathbb{P}$, and with parameters $\left(\tilde{\alpha}, \tilde{C}, \tilde{\lambda}_{-}, \tilde{\lambda}_{+}, r-d\right)$ under a measure $\mathbb{Q}$. Then $\mathbb{Q}$ is an EMM of $\mathbb{P}$ if and only if $\tilde{\alpha}=\alpha, \tilde{C}=C$, and

$$
r-d-\psi_{C G M Y}^{0}\left(-i ; \alpha, \tilde{C}, \tilde{\lambda}_{+}, \lambda_{-}\right)=\mu-\psi_{C G M Y}^{0}\left(-i ; \alpha, C, \lambda_{+}, \lambda_{-}\right) .
$$

If we put $\tilde{\lambda}_{+}=\lambda_{+}-\theta$ and $\tilde{\lambda}_{-}=\lambda_{-}+\theta$, and find $\theta$ satisfying (4), that is

$$
r-d-\psi_{C G M Y}^{0}\left(-i ; \alpha, \lambda_{+}-\theta, \lambda_{-}+\theta, \lambda_{-}\right)=\mu-\psi_{C G M Y}^{0}\left(-i ; \alpha, C, \lambda_{+}, \lambda_{-}\right),
$$

then the CGMY stock price process with parameters $\left(\alpha, C, \lambda_{+}-\theta, \lambda_{-}+\theta, r\right)$ is one risk neutral price process. The measure $\mathbb{Q}^{\theta}$ induced from the driving process of the risk neutral stock price process is the Esscher transformed equivalent martingale measure (EssEMM). The selection of the EssEMM among the set of possible other EMM's is recommended because it maximizes the power utility function (Gerber and Shiu (1996)). 


\section{$3 \quad$ MTS Distributions and MTS Model}

In this section, we will present a variant of the tempered stable distribution named modified tempered stable (MTS) distribution that was introduced in Kim (2005). The proofs can be found in Kim (2005) or Kim, Rachev, and Chung (2006).

Definition 3.1. Let $\alpha \in(0,2) \backslash\{1\}, C, \lambda_{+}, \lambda_{-}>0$, and $m \in \mathbb{R}$. A probability distribution is said to be the modified tempered stable distribution if the characteristic function $\phi_{M T S}$ is given by

$$
\phi_{M T S}\left(u ; \alpha, C, \lambda_{+}, \lambda_{-}, m\right)=\exp \left(i m u+G_{R}\left(u ; \alpha, C, \lambda_{+}, \lambda_{-}\right)+G_{I}\left(u ; \alpha, C, \lambda_{+}, \lambda_{-}\right)\right)
$$

where for $u \in \mathbb{R}$,

$$
\begin{aligned}
& G_{R}\left(u ; \alpha, C, \lambda_{+}, \lambda_{-}\right)=\frac{\sqrt{\pi} C \Gamma\left(-\frac{\alpha}{2}\right)}{2^{\frac{\alpha+3}{2}}}\left(\left(\lambda_{+}^{2}+u^{2}\right)^{\alpha / 2}-\lambda_{+}^{\alpha}+\left(\lambda_{-}^{2}+u^{2}\right)^{\alpha / 2}-\lambda_{-}^{\alpha}\right) \\
& G_{I}\left(u ; \alpha, C, \lambda_{+}, \lambda_{-}\right) \\
& \quad=\frac{i u C \Gamma\left(\frac{1-\alpha}{2}\right)}{2^{\frac{\alpha+1}{2}}}\left(\lambda_{+}^{\alpha-1} F\left(1, \frac{1-\alpha}{2} ; \frac{3}{2} ;-\frac{u^{2}}{\lambda_{+}^{2}}\right)-\lambda_{-}^{\alpha-1} F\left(1, \frac{1-\alpha}{2} ; \frac{3}{2} ;-\frac{u^{2}}{\lambda_{-}^{2}}\right)\right) .
\end{aligned}
$$

Here $F$ is the hypergeometric function. If a random variable $X$ follows the MTS distribution then we denote $X \sim \operatorname{MTS}\left(\alpha, C, \lambda_{+}, \lambda_{-}, m\right)$.

The MTS distribution defined as above is infinitely divisible. Indeed, the characteristic function (6) is obtained by the Lévy-Khintchine formula whose Gaussian part is zero, and the Lévy measure $\nu(d x)$ is equal to

$$
\nu(d x)=C \frac{q_{\alpha}\left(\lambda_{+}|x|\right) 1_{x>0}+q_{\alpha}\left(\lambda_{-}|x|\right) 1_{x<0}}{|x|^{\alpha+1}} d x,
$$

where $q_{\alpha}(x)$ is given by

$$
q_{\alpha}(x)=x^{\frac{\alpha+1}{2}} K_{\frac{\alpha+1}{2}}(x),
$$

and $K_{p}(x)$ is the modified Bessel function of the second kind. The Lévy measure $\nu(d x)$ will be called the MTS Lévy measure with parameters $\left(\alpha, C, \lambda_{+}, \lambda_{-}\right)$. The asymptotic behavior of $\nu(x)$ as follows :

$$
\begin{aligned}
& \nu(x) \sim 2^{\frac{\alpha-1}{2}} C \Gamma\left(\frac{\alpha+1}{2}\right) \frac{1}{|x|^{\alpha+1}}, \quad \text { as } x \rightarrow 0, \\
& \nu(x) \sim \sqrt{\frac{\pi}{2}} C \lambda_{ \pm}^{\frac{\alpha}{2}} \frac{e^{-\lambda_{ \pm} x}}{x^{\frac{\alpha}{2}+1}}, \quad \text { as } x \rightarrow \pm \infty .
\end{aligned}
$$


That is, $\nu(x)$ behaves like an $\alpha$-stable distribution near zero, and it has exponential decay at the tails.

Proposition 3.2. Let $X \sim \operatorname{MTS}\left(\alpha, C, \lambda_{+}, \lambda_{-}, m\right)$. Then the exponential moment $E\left[e^{u X}\right]$ is finite if $-\lambda_{-}<u<\lambda_{+}$. Furthermore, if $|u|<\lambda_{+} \wedge \lambda_{-}$then it is given by

$$
E\left[e^{u X}\right]=\exp \left(m u+G_{R}\left(-i u ; \alpha, C, \lambda_{+}, \lambda_{-}\right)+G_{I}\left(-i u ; \alpha, C, \lambda_{+}, \lambda_{-}\right)\right) .
$$

Using the characteristic function (6), we obtain the cumulants of all orders. The cumulants $c_{n}(X)$ of $X \sim \operatorname{MTS}\left(\alpha, C, \lambda_{+}, \lambda_{-}, m\right)$ are given as follows (see Proposition 3.4.1 in Kim $(2005))$ :

$$
\begin{aligned}
& c_{n}(X) \\
& = \begin{cases}m \cdot 1_{n=1}+2^{n-\frac{\alpha+3}{2}}\left(\frac{n-1}{2}\right) ! C \Gamma\left(\frac{n-\alpha}{2}\right)\left(\lambda_{+}^{\alpha-n}-\lambda_{-}^{\alpha-n}\right) & \text { if } n=1,3,5, \cdots \\
2^{-\frac{\alpha+3}{2}} \sqrt{\pi} \frac{n !}{\left(\frac{n}{2}\right) !} C \Gamma\left(\frac{n-\alpha}{2}\right)\left(\lambda_{+}^{\alpha-n}+\lambda_{-}^{\alpha-n}\right) & \text { if } n=2,4,6 \cdots\end{cases}
\end{aligned}
$$

Moreover, we obtain the mean, variance, skewness, and excess kurtosis using the cumulants.

$$
\begin{gathered}
E[X]=c_{1}(X)=m+2^{-\frac{\alpha+1}{2}} C \Gamma\left(\frac{1-\alpha}{2}\right)\left(\lambda_{+}^{\alpha-1}-\lambda_{-}^{\alpha-1}\right) \\
\operatorname{Var}(X)=c_{2}(X)=2^{-\frac{\alpha+1}{2}} \sqrt{\pi} C \Gamma\left(1-\frac{\alpha}{2}\right)\left(\lambda_{+}^{\alpha-2}+\lambda_{-}^{\alpha-2}\right) \\
s(X)=\frac{c_{3}(X)}{c_{2}(X)^{\frac{3}{2}}}=\frac{2^{\frac{\alpha+9}{4}} \Gamma\left(\frac{3-\alpha}{2}\right)\left(\lambda_{+}^{\alpha-3}-\lambda_{-}^{\alpha-3}\right)}{\pi^{\frac{3}{4}} C^{\frac{1}{2}}\left(\Gamma\left(1-\frac{\alpha}{2}\right)\left(\lambda_{+}^{\alpha-2}+\lambda_{-}^{\alpha-2}\right)\right)^{\frac{3}{2}}} \\
k(X)=\frac{c_{4}(X)}{c_{2}(X)^{2}}=\frac{\left.3 \cdot 2^{\frac{\alpha+3}{2}} \Gamma\left(2-\frac{\alpha}{2}\right)\left(\lambda_{+}^{\alpha-4}+\lambda_{-}^{\alpha-4}\right)\right)}{\sqrt{\pi} C\left(\Gamma\left(1-\frac{\alpha}{2}\right)\left(\lambda_{+}^{\alpha-2}+\lambda_{-}^{\alpha-2}\right)\right)^{2}}
\end{gathered}
$$

The parameters $\lambda_{+}$and $\lambda_{-}$control the rate of decay on the positive and negative part, respectively. If $\lambda_{+}>\lambda_{-}\left(\lambda_{+}<\lambda_{-}\right)$, then the distribution is skewed to the left (right). Moreover, if $\lambda_{+}=\lambda_{-}$, then it is symmetric. If we put

$$
\begin{aligned}
& C\left(\alpha, \lambda_{+}, \lambda_{-}\right)=2^{\frac{\alpha+1}{2}}\left(\sqrt{\pi} \Gamma\left(1-\frac{\alpha}{2}\right)\left(\lambda_{+}^{\alpha-2}+\lambda_{-}^{\alpha-2}\right)\right)^{-1} \\
& m\left(\alpha, \lambda_{+}, \lambda_{-}\right)=-\frac{\Gamma\left(\frac{1-\alpha}{2}\right)\left(\lambda_{+}^{\alpha-1}-\lambda_{-}^{\alpha-1}\right)}{\sqrt{\pi} \Gamma\left(1-\frac{\alpha}{2}\right)\left(\lambda_{+}^{\alpha-2}+\lambda_{-}^{\alpha-2}\right)}
\end{aligned}
$$

then $X \sim \operatorname{MTS}\left(\alpha, C\left(\alpha, \lambda_{+}, \lambda_{-}\right), \lambda_{+}, \lambda_{-}, m\left(\alpha, \lambda_{+}, \lambda_{-}\right)\right)$has zero mean and unit variance, and $X$ will be called the standard MTS distribution and denoted by $X \sim \operatorname{stdMTS}\left(\alpha, \lambda_{+}\right.$, $\left.\lambda_{-}\right)$. Moreover, the log-Laplace transform of $X$ will be denoted by $L_{M T S}\left(x ; \alpha, \lambda_{+}, \lambda_{-}\right)$. By 
Proposition 3.2, the function $L_{M T S}\left(x ; \alpha, \lambda_{+}, \lambda_{-}\right)$is finite if $x \in\left(-\lambda_{-}, \lambda_{+}\right)$, and if $|x|<\lambda_{+} \wedge \lambda_{-}$ then we have

$$
\begin{array}{rl}
L_{M T S}\left(x ; \alpha, \lambda_{+}, \lambda_{-}\right)=x & m\left(\alpha, \lambda_{+}, \lambda_{-}\right)+G_{R}\left(-i x ; \alpha, C\left(\alpha, \lambda_{+}, \lambda_{-}\right), \lambda_{+}, \lambda_{-}\right) \\
+ & G_{I}\left(-i x ; \alpha, C\left(\alpha, \lambda_{+}, \lambda_{-}\right), \lambda_{+}, \lambda_{-}\right) .
\end{array}
$$

Since the MTS distribution is infinitely divisible, we can generate a Lévy process called the MTS process.

Definition 3.3. A Lévy process $X=\left(X_{t}\right)_{t \geq 0}$ is said to be a MTS process with parameters $\left(\alpha, C, \lambda_{+}, \lambda_{-}, m\right)$ if $X_{1} \sim \operatorname{MTS}\left(\alpha, C, \lambda_{+}, \lambda_{-}, m\right)$.

The parameter $\alpha$ determines the path behavior; that is, the MTS process has finite variation if $\alpha<1$ and infinite variation if $\alpha>1$ (see Proposition 3.5.4 in Kim (2005)). The following theorem will be used for determining the EMM.

Proposition 3.4. Suppose $\left(X_{t}\right)_{t \in[0, T]}$ is the MTS process with parameters $\left(\alpha, C, \lambda_{+}, \lambda_{-}, m\right)$ under $\mathbb{P}$, and the MTS process with parameters $\left(\tilde{\alpha}, \tilde{C}, \tilde{\lambda}_{+}, \tilde{\lambda}_{-}, \tilde{m}\right)$ under $\mathbb{Q}$. Then $\left.\mathbb{P}\right|_{\mathcal{F}_{t}}$ and $\left.\mathbb{Q}\right|_{\mathcal{F}_{t}}$ are equivalent for all $t>0$ if and only if $C=\tilde{C}, \alpha=\tilde{\alpha}$, and $\tilde{m}=m$.

Applying Proposition 3.4 to MTS distributed random variables, we can obtain the following corollary.

Corollary 3.5. (a) Let $X \sim \operatorname{MTS}\left(\alpha, C, \lambda_{+}, \lambda_{-}, m\right)$ under a measure $\mathbf{P}$, and $X \sim \operatorname{MTS}(\tilde{\alpha}$, $\left.\tilde{C}, \tilde{\lambda}_{+}, \tilde{\lambda}_{-}, \tilde{m}\right)$ under a measure $\mathbf{Q}$. Then $\mathbf{P}$ and $\mathbf{Q}$ are equivalent if and only if $C=\tilde{C}$, $\alpha=\tilde{\alpha}$, and $\tilde{m}=m$.

(b) Let $X \sim \operatorname{stdMTS}\left(\alpha, \lambda_{+}, \lambda_{-}\right)$under a measure $\mathbf{P}$, and $(X+k) \sim \operatorname{stdMTS}\left(\tilde{\alpha}, \tilde{\lambda}_{+}, \tilde{\lambda}_{-}\right)$ under a measure $\mathbf{Q}$ for a constant $k \in \mathbb{R}$. Then $\mathbf{P}$ and $\mathbf{Q}$ are equivalent if and only if

$$
\left(\begin{array}{l}
\alpha=\tilde{\alpha}, \\
\tilde{\lambda}_{+}^{\alpha-2}+\tilde{\lambda}_{-}^{\alpha-2}=\lambda_{+}^{\alpha-2}+\lambda_{-}^{\alpha-2} \\
k=\frac{\Gamma\left(\frac{1-\alpha}{2}\right)\left(\lambda_{+}^{\alpha-1}-\lambda_{-}^{\alpha-1}-\tilde{\lambda}_{+}^{\alpha-1}+\tilde{\lambda}_{-}^{\alpha-1}\right)}{\sqrt{\pi} \Gamma\left(1-\frac{\alpha}{2}\right)\left(\tilde{\lambda}_{+}^{\alpha-2}+\tilde{\lambda}_{-}^{\alpha-2}\right)} .
\end{array}\right.
$$

In the continuous-time market, the MTS model will be defined as follows:

Definition 3.6. Let $\alpha \in(0,2) \backslash\{1\}, C, \lambda_{+}, \lambda_{-}>0$, and $\mu \in \mathbb{R}$. In the continuous-time market, if the driving process $\left(X_{t}\right)_{t \in[0, T]}$ of $\left(S_{t}\right)_{t \in[0, T]}$ is the MTS process with parameters 
$\left(\alpha, C, \lambda_{+}, \lambda_{-}, m\right)$ and $m=\mu-\log \phi_{M T S}\left(-i ; \alpha, C, \lambda_{+}, \lambda_{-}, 0\right)$, then the stock price process $\left(S_{t}\right)_{t \in[0, T]}$ is called the MTS stock price process and we say that the stock price process follows the MTS model.

By Theorem 3.4, we can prove the following theorem. For convenience, let us define

$$
\psi_{M T S}^{0}\left(u ; \alpha, C, \lambda_{+}, \lambda_{-}\right)=G_{R}\left(u ; \alpha, C, \lambda_{+}, \lambda_{-}\right)+G_{I}\left(u ; \alpha, C, \lambda_{+}, \lambda_{-}\right)
$$

Theorem 3.7 (EMM conditions for the MTS model). Assume that $\left(S_{t}\right)_{t \in[0, T]}$ is the MTS stock price process with parameters $\left(\alpha, C, \lambda_{+}, \lambda_{-}, \mu\right)$ under the market measure $\mathbb{P}$, and with parameters $\left(\tilde{\alpha}, \tilde{C}, \tilde{\lambda}_{+}, \tilde{\lambda}_{-}, r-d\right)$ under a measure $\mathbb{Q}$. Then $\mathbb{Q}$ is an EMM of $\mathbb{P}$ if and only if $C=\tilde{C}, \alpha=\tilde{\alpha}$ and

$$
r-d-\psi_{M T S}^{0}\left(-i ; \alpha, C, \lambda_{+}, \lambda_{-}\right)=\mu-\psi_{M T S}^{0}\left(-i ; \alpha, C, \tilde{\lambda}_{+}, \tilde{\lambda}_{-}\right)
$$

\section{$4 \quad$ KR Tempered Stable Distribution}

In this section, we present the KR tempered stable distribution. The proofs can be found in Kim et al. (2007).

Definition 4.1. Let $\alpha \in(0,2) \backslash\{1\}, k_{+}, k_{-}, r_{+}, r_{-} \in(0, \infty), p_{+}, p_{-} \in(-\alpha, \infty) \backslash\{-1,0\}$, and $m \in \mathbb{R}$. A tempered stable distribution is said to be the $K R$ tempered stable distribution (simply the $K R$ distribution) if its characteristic function $\phi_{K R}$ is given by

$$
\phi_{K R}\left(u ; \alpha, k_{+}, k_{-}, r_{+}, r_{-}, p_{+}, p_{-}, m\right)=\exp \left(i u m+\psi_{K R}^{0}\left(u ; \alpha, k_{+}, k_{-}, r_{+}, r_{-}, p_{+}, p_{-}\right)\right)
$$

where

$$
\begin{aligned}
& \psi_{K R}^{0}\left(u ; \alpha, k_{+}, k_{-}, r_{+}, r_{-}, p_{+}, p_{-}\right) \\
& =H_{\alpha}\left(u ; k_{+}, r_{+}, p_{+}\right)+H_{\alpha}\left(-u ; k_{-}, r_{-}, p_{-}\right)+i u \alpha \Gamma(-\alpha)\left(\frac{k_{+} r_{+}}{p_{+}+1}-\frac{k_{-} r_{-}}{p_{-}+1}\right),
\end{aligned}
$$

and

$$
H_{\alpha}(u ; x, y, p)=\frac{x \Gamma(-\alpha)}{p}(F(p,-\alpha ; 1+p ; i y u)-1) .
$$

Here $F$ is the hypergeometric function. If a random variable $X$ follows the $K R$ distribution, then we denote $X \sim \operatorname{KR}\left(\alpha, k_{+}, k_{-}, r_{+}, r_{-}, p_{+}, p_{-}, m\right)$. 
The KR distribution can be constructed as follows. Consider a spectral measure $R$ as

$$
R(d x)=\left(k_{+} r_{+}^{-p_{+}} I_{x \in\left(0, r_{+}\right)}|x|^{p_{+}-1}+k_{-} r_{-}^{-p_{-}} I_{x \in\left(-r_{-}, 0\right)}|x|^{p_{-}-1}\right) d x .
$$

Then there is a Lévy measure $M$ corresponding to $R$ and $M$ can be written in the form

$$
\begin{aligned}
M(A)= & k_{+} r_{+}^{-p_{+}} \int_{0}^{r_{+}} \int_{0}^{\infty} I_{A}(t x) t^{-\alpha-1} e^{-t} d t x^{p_{+}-1} d x \\
& +k_{-} r_{-}^{-p_{-}} \int_{0}^{r_{-}} \int_{0}^{\infty} I_{A}(-t x) t^{-\alpha-1} e^{-t} d t x^{p_{-}-1} d x, \quad A \in \mathcal{B}\left(\mathbb{R}_{0}\right)
\end{aligned}
$$

by Theorem 2.3 of Rosiński (2006). By Theorem 2.9 in Rosiński (2006), we can obtain the characteristic function (15). The KR distribution has the exponential moment as follows:

Proposition 4.2. Let $X \sim \operatorname{KR}\left(\alpha, k_{+}, k_{-}, r_{+}, r_{-}, p_{+}, p_{-}, m\right)$. Then $E\left[e^{\theta X}\right]<\infty$ if and only if $-r_{-}^{-1} \leq \theta \leq r_{+}^{-1}$, and in this case we have

$$
E\left[e^{\theta X}\right]=\phi_{K R}\left(-i \theta ; \alpha, k_{+}, k_{-}, r_{+}, r_{-}, p_{+}, p_{-}, m\right)
$$

If $X \sim \operatorname{KR}\left(\alpha, k_{+}, k_{-}, r_{+}, r_{-}, p_{+}, p_{-}, m\right)$, then the cumulants of $X$ are given by $c_{1}(X)=m$ and

$$
c_{n}(X)=\Gamma(n-\alpha)\left(\frac{k_{+} r_{+}^{n}}{p_{+}+n}+(-1)^{n} \frac{k_{-} r_{-}^{n}}{p_{-}+n}\right), \quad n \geq 2 .
$$

Hence, we obtain the mean, variance, skewness, and excess kurtosis of $X$ which are given as follows:

$$
\begin{aligned}
& E[X]=c_{1}(X)=m \\
& \operatorname{Var}(X)=c_{2}(X)=\Gamma(2-\alpha)\left(\frac{k_{+} r_{+}^{2}}{p_{+}+2}+\frac{k_{-} r_{-}^{2}}{p_{-}+2}\right) \\
& s(X)=\frac{c_{3}(X)}{c_{2}(X)^{3 / 2}}=\frac{\Gamma(3-\alpha)\left(\frac{k_{+} r_{+}^{3}}{p_{+}+3}-\frac{k_{-} r_{-}^{3}}{p_{-}+3}\right)}{\Gamma(2-\alpha)^{3 / 2}\left(\frac{k_{+} r_{+}^{2}}{p_{+}+2}+\frac{k_{-} r_{-}^{2}}{p_{-}+2}\right)^{3 / 2}} \\
& k(X)=\frac{c_{4}(X)}{c_{2}(X)^{2}}=\frac{\Gamma(4-\alpha)\left(\frac{k_{+} r_{+}^{4}}{p_{+}+4}+\frac{k_{-} r_{-}^{4}}{p_{-}+4}\right)}{\Gamma(2-\alpha)^{2}\left(\frac{k_{+} r_{+}^{2}}{p_{+}+2}+\frac{k_{-} r_{-}^{2}}{p_{-}+2}\right)^{2}} .
\end{aligned}
$$

If we put

$$
k_{+}\left(\alpha, r_{+}, p_{+}\right)=\frac{p_{+}+2}{2 \Gamma(2-\alpha) r_{+}^{2}}, \quad k_{-}\left(\alpha, r_{-}, p_{-}\right)=\frac{p_{-}+2}{2 \Gamma(2-\alpha) r_{-}^{2}},
$$

then $X \sim \operatorname{KR}\left(\alpha, k_{+}\left(\alpha, r_{+}, p_{+}\right), k_{-}\left(\alpha, r_{-}, p_{-}\right), r_{+}, r_{-}, p_{+}, p_{-}, 0\right)$ has zero mean and unit variance. In this case, $X$ is said to be standard $K R$ tempered stable distributed (or standard KR 
distributed $)$ and denote $X \sim \operatorname{StdKR}\left(\alpha, r_{+}, r_{-}, p_{+}, p_{-}\right)$. Moreover, the log-Laplace transform of $X$ will be denoted by $L_{K R}\left(x ; \alpha, r_{+}, r_{-}, p_{+}, p_{-}\right)$which is finite in $x \in\left(-1 / r_{-}, 1 / r_{+}\right)$, and we can obtain

$$
\begin{aligned}
& L_{K R}\left(x ; \alpha, r_{+}, r_{-}, p_{+}, p_{-}\right) \\
& =\psi_{K R}^{0}\left(-i x ; \alpha, k_{+}\left(\alpha, r_{+}, p_{+}\right), k_{-}\left(\alpha, r_{-}, p_{-}\right), r_{+}, r_{-}, p_{+}, p_{-}, 0\right)
\end{aligned}
$$

where $\psi_{K R}^{0}$ is given by $(16)$.

The KR distribution is an extension of the CGMY distribution.

Proposition 4.3. The distribution $\operatorname{KR}\left(\alpha, k_{+}, k_{-}, r_{+}, r_{-}, p_{+}, p_{-}, m\right)$ converges weakly to the $C G M Y$ distribution as $p_{ \pm} \rightarrow \infty$ provided that $k_{ \pm}=c\left(\alpha+p_{ \pm}\right) r_{ \pm}^{-\alpha}$ where $c>0$

Figure 1 shows that the KR distributions converge to the CGMY distribution when parameter $p=p_{+}=p_{-}$increases.

Since the KR distribution is infinitely divisible, a Lévy process can be induced from the distribution.

Definition 4.4. A Lévy process $X=\left(X_{t}\right)_{t \geq 0}$ is said to be a KR tempered stable process or simply a KR process with parameters $\left(\alpha, k_{+}, k_{-}, r_{+}, r_{-}, p_{+}, p_{-}, m\right)$ if $X_{1} \sim$ $\mathrm{KR}\left(\alpha, k_{+}, k_{-}, r_{+}, r_{-}, p_{+}, p_{-}, m\right)$.

The KR process $\left(X_{t}\right)_{t \geq 0}$ with parameters $\left(\alpha, k_{+}, k_{-}, r_{+}, r_{-}, p_{+}, p_{-}, m\right)$ has finite variation if $\alpha \in(0,1)$ and infinite variation if $\alpha \in(1,2)$ (see Proposition 3.12 in Kim et al. (2007)).

Just as in the MTS case, we need the following theorem for finding the EMM:

Theorem 4.5. Consider two probability measure $\mathbb{P}_{1}, \mathbb{P}_{2}$ and the canonical process $\left(X_{t}\right)_{t \geq 0}$ on $\left(\Omega, \mathcal{F},\left(\mathcal{F}_{t}\right)_{t \geq 0}\right)$ given above. For each $j=1,2$, suppose $\left(X_{t}\right)_{t \geq 0}$ is the KR tempered stable process under $\mathbb{P}_{j}$ with parameters $\left(\alpha_{j}, k_{j,+}, k_{j,-}, r_{j,+}, r_{j,-}, p_{j,+}, p_{j,-}, m_{j}\right)$ and

$$
\left\{\begin{array}{ll}
p_{j, \pm} \in\left(1 / 2-\alpha_{j}, \infty\right) \backslash\{0\}, & \alpha_{j} \in(0,1) \\
p_{j, \pm} \in\left(1-\alpha_{j}, \infty\right) \backslash\{0\}, & \alpha_{j} \in(1,2)
\end{array} .\right.
$$

Then $\left.\mathbb{P}_{1}\right|_{\mathcal{F}_{t}}$ and $\left.\mathbb{P}_{2}\right|_{\mathcal{F}_{t}}$ are equivalent for every $t>0$ if and only if the parameters satisfy the 
condition

$$
\left(\begin{array}{l}
\alpha:=\alpha_{1}=\alpha_{2} \\
\frac{k_{1,+} r_{1,+}^{\alpha}}{\alpha+p_{1,+}}=\frac{k_{2,+} r_{2,+}^{\alpha}}{\alpha+p_{2,+}} \\
\frac{k_{1,-} r_{1,-}^{\alpha}}{\alpha+p_{1,-}}=\frac{k_{2,-} r_{2,-}^{\alpha}}{\alpha+p_{2,-}} \\
m_{2}-m_{1}=\Gamma(1-\alpha) \sum_{j=1,2}(-1)^{j}\left(\frac{k_{j,+} r_{j,+}}{p_{j,+}+1}-\frac{k_{j,-} r_{j,-}}{p_{j,-}+1}\right)
\end{array}\right.
$$

Applying Theorem 4.5 to KR distributed random variables, we obtain the following corollary.

Corollary 4.6. Assume that $\alpha_{1}, \alpha_{2} \in(0,2) \backslash\{1\}$, and $p_{1,+}, p_{1,-}, p_{2,+}$, and $p_{2,-}$ satisfy the condition (20).

(a) For each $j=1,2$, suppose $X \sim \mathrm{KR}\left(\alpha_{j}, k_{j,+}, k_{j,-}, r_{j,+}, r_{j,-}, p_{j,+}, p_{j,-}, m_{j}\right)$ under a measure $\mathbf{P}_{j}$. Then $\mathbf{P}_{1}$ and $\mathbf{P}_{2}$ are equivalent if and only if the parameters satisfy the condition (21).

(b) For a constant $k \in \mathbb{R}$, suppose $X \sim \operatorname{stdKR}\left(\alpha_{1}, r_{1,+}, r_{1,-}, p_{1,+}, p_{1,-}\right)$ and $(X+k) \sim$ $\operatorname{stdKR}\left(\alpha_{2}, r_{2,+}, r_{2,-}, p_{2,+}, p_{2,-}\right)$ under measures $\mathbf{P}_{1}$ and $\mathbf{P}_{2}$, respectively. Then $\mathbf{P}_{1}$ and $\mathbf{P}_{2}$ are equivalent if and only if the parameters satisfy the condition

$$
\left(\begin{array}{l}
\alpha:=\alpha_{1}=\alpha_{2} \\
\frac{p_{2,+}+2}{p_{2,+}+\alpha} r_{2,+}^{\alpha-2}=\frac{p_{1,+}+2}{p_{1,+}+\alpha} r_{1,+}^{\alpha-2} \\
\frac{p_{2,-}+2}{p_{2,-}+\alpha} r_{2,-}^{\alpha-2}=\frac{p_{1,-}+2}{p_{1,-}+\alpha} r_{1,-}^{\alpha-2} \\
k=\frac{\Gamma(1-\alpha)}{2 \Gamma(2-\alpha)} \sum_{j=1,2}(-1)^{j}\left(\frac{p_{j,+}+2}{r_{j,+}\left(p_{j,+}+1\right)}-\frac{p_{j,-}+2}{r_{j,-}\left(p_{j,-}+1\right)}\right)
\end{array}\right.
$$

\subsection{KR Model}

Recall the continuous-time market $\left(\Omega, \mathcal{F}_{T},\left(\mathcal{F}_{t}\right)_{t \in[0, T]}, \mathbb{P}\right)$, the driving process $\left(X_{t}\right)_{t \in[0, T]}$, the market price process $\left(S_{t}\right)_{t \in[0, T]}$, and the EMM $\mathbb{Q}$ from the preliminary.

Definition 4.7. In the continuous-time market, if $\left(X_{t}\right)_{t \in[0, T]}$ is the KR process with parameters $\left(\alpha, k_{+}, k_{-}, r_{+}, r_{-}, p_{+}, p_{-}, m\right)$ where $\alpha \in(0,1) \cup(1,2), k_{+}, k_{-}, r_{-} \in(0, \infty), r_{+} \in(0,1)$,

$$
\left\{\begin{array}{ll}
p_{+}, p_{-} \in(1 / 2-\alpha, \infty) \backslash\{0\}, & \text { if } \quad \alpha \in(0,1), \\
p_{+}, p_{-} \in(1-\alpha, \infty) \backslash\{0\}, & \text { if } \quad \alpha \in(1,2)
\end{array},\right.
$$

and $m=\mu-\psi_{K R}^{0}\left(-i ; \alpha, k_{+}, k_{-}, r_{+}, r_{-}, p_{+}, p_{-}\right)$for some $\mu \in \mathbb{R}$, then the process $\left(S_{t}\right)_{t \in[0, T]}$ is called the KR price process with parameters $\left(\alpha, k_{+}, k_{-}, r_{+}, r_{-}, p_{+}, p_{-}, \mu\right)$ and we say that the stock price process follows the KR model. 
Remark 4.8. We have the condition $r_{+} \in(0,1)$ for $\psi_{K R}^{0}\left(-i ; \alpha, k_{+}, k_{-}, r_{+}, r_{-}, p_{+}, p_{-}\right)$to be well defined. By the condition (23), we are able to use Theorem 4.5 for finding an EMM. Since $m=\mu-\psi_{K R}^{0}\left(-i ; \alpha, k_{+}, k_{-}, r_{+}, r_{-}, p_{+}, p_{-}\right)$, we have

$$
E\left[S_{t}\right]=S_{0} E\left[e^{X_{t}}\right]=S_{0} e^{\mu t}
$$

Theorem 4.9 (EMM conditions for the KR model). Assume that $\left(S_{t}\right)_{t \in[0, T]}$ is the the KR price process with parameters $\left(\alpha, k_{+}, k_{-}, r_{+}, r_{-}, p_{+}, p_{-}, \mu\right)$ under the market measure $\mathbb{P}$, and with parameters $\left(\tilde{\alpha}, \tilde{a}_{+}, \tilde{a}_{-}, \tilde{r}_{+}, \tilde{r}_{-}, \tilde{p}_{+}, \tilde{p}_{-}, r-d\right)$ under a measure $\mathbb{Q}$. Then $\mathbb{Q}$ is an EMM of $\mathbb{P}$ if and only if

$$
\left(\begin{array}{rl}
\alpha=\tilde{\alpha} & \tilde{k}_{+} r_{+}^{\alpha} \\
\frac{\tilde{k}_{+} \tilde{r}_{+}^{\alpha}}{\alpha+p_{+}} & =\frac{\tilde{p}_{+}}{\alpha+\tilde{p}_{+}} \\
\frac{k_{-} r_{-}^{\alpha}}{\alpha+p_{-}}= & \frac{\tilde{k}_{-} \tilde{r}_{-}^{\alpha}}{\alpha+\tilde{p}_{-}} \\
\mu-(r-d) \stackrel{H_{\alpha}\left(-i ; k_{+}, r_{+}, p_{+}\right)+H_{\alpha}\left(i ; k_{-}, r_{-}, p_{-}\right)}{=} & \quad-H_{\alpha}\left(-i ; \tilde{k}_{+}, \tilde{r}_{+}, \tilde{p}_{+}\right)-H_{\alpha}\left(i ; \tilde{k}_{-}, \tilde{r}_{-}, \tilde{p}_{-}\right)
\end{array}\right.
$$

Proof. By Definition 4.7 and Theorem 4.5, it can be proved.

\section{Estimation of the Parameters for the Exponential Tempered Stable Models}

We estimate the market parameters and the risk-neutral parameters of the CGMY, MTS, and KR model for the closing price of the S\&P 500 index. Data were supplied by Option Metrics's IvyDB in the Wharton Research Data Services. We select 10 mid-month Wednesdays (June 8, 2005; July 13, 2005; August 10, 2005; September 14, 2005; October 12, 2005; November 9, 2005; December 14, 2005; January 11, 2006; February 8, 2006; March 15, 2006) and estimate the market and risk-neutral parameters of the index price process. We first estimate the market parameters of each model from 10 years of time-series data, and then we estimate the risk-neutral parameters using prices of the S\&P 500 option with maturities between 1 and 2 months for each Wednesday. The market option prices are computed by using the Black-Scholes formula with the implied volatilities and dividends given by IvyDB.

We use the maximum likelihood method (MLE) for estimating the market parameters. The density functions of each model are obtained by the discrete Fourier transform method. For each model, we estimate the risk-neutral parameters using the method of least-squares 
calibration with a prior; that is, we estimate the risk-neutral parameters by nonlinear least squares minimization under the EMM conditions of Theorem 2.6, Theorem 3.7, and Theorem 4.9. The CGMY and MTS models have four risk-neutral parameters to be estimated and three restrictions in their EMM conditions, while the exponential KR model has seven riskneutral parameters and four restrictions. Hence the CGMY and MTS models have only one free parameter, while the $\mathrm{KR}$ model has three free parameters for the estimation. For the calculation of the model's option price, we followed the Fourier transform method of Carr and Madan (1999) and Lewis (2001).

We use the Kolmogrov-Smirnov (KS) statistic and its p-values for the assessment of the goodness of fit. Table 1 gives the estimated market parameters and their KS statistic and $p$-values for each time series and each model. No estimated market parameters are rejected and have good $p$-values. Moreover, the $p$-values of the KS statistic for the KR model exceed the $p$-values of the CGMY and MTS models.

The risk-neutral parameters for given market parameters of the selected mid-month Wednesdays are shown in Table 2. We compare the result of the risk-neutral parameter estimation using the error estimators, absolute percentage error (APE), average absolute error (AAE), and root mean square error (RMSE). These are defined as follows (Schoutens $(2003))$ :

$$
\begin{aligned}
\mathrm{AAE} & =\sum_{\text {options }} \frac{\mid \text { market price }- \text { model price } \mid}{\text { number of options }} \\
\mathrm{APE} & =\frac{1}{\text { mean option price }} \sum_{\text {options }} \frac{\mid \text { market price }- \text { model price } \mid}{\text { number of options }}, \\
\mathrm{RMSE} & =\sqrt{\sum_{\text {options }} \frac{(\text { market price }- \text { model price })^{2}}{\text { number of options }}} .
\end{aligned}
$$

The error estimators of the KR distributed EMM are less than those of the CGMY and MTS parameter fits. The relatively flexible change of measure for the KR distribution seems to generate the good performance. 


\section{GARCH Model with Infinitely Divisible Distributed Innovations}

In this section we will present a new GARCH model with the infinitely divisible distributed innovation process. The new GARCH stock price model is defined over a filtered probability space $\left(\Omega, \mathfrak{F},\left(\mathfrak{F}_{t}\right)_{t \in \mathbb{N}}, \mathbb{P}\right)$ which is constructed as follows: Consider a sequence $\left(\varepsilon_{t}\right)_{t \in \mathbb{N}}$ of iid real random variables on a sequence of probability spaces $\left(\Omega_{t}, \mathbf{P}_{t}\right)_{t \in \mathbb{N}}$, such that $\varepsilon_{t}$ is an infinitely divisible distributed random variable with zero mean and unit variance on $\left(\Omega_{t}, \mathbf{P}_{t}\right)$, and assume that $E\left[e^{x \varepsilon_{t}}\right]<\infty$ where $x \in(-a, b)$ for some $a, b>0$. Now we define $\Omega:=\prod_{t \in \mathbb{N}} \Omega_{t}$, $\mathfrak{F}_{t}:=\otimes_{k=1}^{t} \sigma\left(\varepsilon_{k}\right) \otimes \mathfrak{F}_{0} \otimes \mathfrak{F}_{0} \cdots, \mathfrak{F}:=\sigma\left(\cup_{t \in \mathbb{N}} \mathfrak{F}_{t}\right)$, and $\mathbb{P}:=\otimes_{t \in \mathbb{N}} \mathbf{P}_{t}$, where $\mathfrak{F}_{0}=\{\emptyset, \Omega\}$ and $\sigma\left(\varepsilon_{k}\right)$ means the $\sigma$-algebra generated by $\varepsilon_{k}$ on $\Omega_{k}$.

We first propose the following stock price dynamics:

$$
\log \left(\frac{S_{t}}{S_{t-1}}\right)=r_{t}-d_{t}+\lambda_{t} \sigma_{t}-L\left(\sigma_{t}\right)+\sigma_{t} \varepsilon_{t}, \quad t \in \mathbb{N},
$$

where $S_{t}$ is the stock price at time $t, r_{t}$ and $d_{t}$ denote the risk-free and dividend rate for the period $[t-1, t]$, respectively, and $\lambda_{t}$ is a $\mathfrak{F}_{t-1}$ measurable random variable. $S_{0}$ is the present observed price. The function $L(x)$ is the $\log$-Laplace-transform of $\varepsilon_{t}$, i.e, $L(x)=\log \left(E\left[e^{x \varepsilon_{t}}\right]\right)$, which is defined on the interval $(-a, b)$. The one period ahead conditional variance $\sigma_{t}^{2}$ follows a $\operatorname{GARCH}(1,1)$ process with a restriction $0<\sigma_{t}<b$, i.e,

$$
\sigma_{t}^{2}=\left(\alpha_{0}+\alpha_{1} \sigma_{t-1}^{2} \varepsilon_{t-1}^{2}+\beta_{1} \sigma_{t-1}^{2}\right) \wedge \rho, \quad t \in \mathbb{N}, \quad \varepsilon_{0}=0
$$

where $\alpha_{0}, \alpha_{1}$ and $\beta_{1}$ are non-negative, $\alpha_{1}+\beta_{1}<1, \alpha_{0}>0$, and $0<\rho<b^{2}$. Clearly the process $\left(\sigma_{t}\right)_{t \in \mathbb{N}}$ is predictable. Moreover, the conditional expectation

$$
E\left[e^{d_{t}} S_{t} / S_{t-1} \mid \mathfrak{F}_{t-1}\right]=\exp \left(r_{t}+\lambda_{t} \sigma_{t}\right)
$$

Thus $\lambda_{t}$ can be interpreted as the market price of risk.

The class of the tempered stable distributions having the exponential moments is a subclass of the infinitely divisible distribution, and suitable for constructing the new GARCH model having infinitely divisible distributed innovation. The stock price dynamics defined as (25) with the conditional variance defined as $(26)$ over the probability space $\left(\Omega, \mathfrak{F},\left(\mathfrak{F}_{t}\right)_{t \in \mathbb{N}}\right.$, $\mathbb{P})$, where $\left(\varepsilon_{t}\right)_{t \in \mathbb{N}}$ is the sequence of tempered stable distributed iid real random variables, 
is called the tempered stable GARCH model. If $\varepsilon_{t}$ equals the standard normal distributed random variable for all $t \in \mathbb{N}$ then $L(x)$ is defined on the whole real line. Consequently, we can ignore the restriction $\sigma_{t}<b$ and hence the model becomes "the normal-GARCH model" introduced by Duan (1995).

\subsection{CTS-GARCH Model}

Consider the tempered stable GARCH model with the sequence $\left(\varepsilon_{t}\right)_{t \in \mathbb{N}}$ of iid random variables with $\varepsilon_{t} \sim \operatorname{stdCTS}\left(\alpha, \lambda_{+}, \lambda_{-}\right)$for all $t \in \mathbb{N}$. We will call the tempered stable GARCH model the CTS-GARCH model. Since $E\left[e^{x \varepsilon_{t}}\right]<\infty$ if $x \in\left(-\lambda_{-}, \lambda_{+}\right), \rho$ has to be in the interval $\left(0, \lambda_{+}{ }^{2}\right)$.

By Corollary 2.4 (b), we can prove the following proposition.

Proposition 6.1. Consider the CTS-GARCH model. Let $T \in \mathbb{N}$ be a time horizon, fix natural number $t \leq T$. Suppose $\tilde{\lambda}_{+}(t)$ and $\tilde{\lambda}_{-}(t)$ satisfy the following conditions:

$$
\left(\begin{array}{l}
\tilde{\lambda}_{+}(t)^{2}>\rho \\
\tilde{\lambda}_{+}(t)^{\alpha-2}+\tilde{\lambda}_{-}(t)^{\alpha-2}=\lambda_{+}{ }^{\alpha-2}+\lambda_{-}{ }^{\alpha-2} \\
\frac{\lambda_{+}{ }^{\alpha-1}-\lambda_{-}{ }^{\alpha-1}-\tilde{\lambda}_{+}(t)^{\alpha-1}+\tilde{\lambda}_{-}(t)^{\alpha-1}}{(1-\alpha)\left(\lambda_{+}{ }^{\alpha-2}+\lambda_{-}{ }^{\alpha-2}\right)} \\
\quad=\lambda_{t}+\frac{1}{\sigma_{t}}\left(L_{C T S}\left(\sigma_{t} ; \alpha, \tilde{\lambda}_{+}(t), \tilde{\lambda}_{-}(t)\right)-L_{C T S}\left(\sigma_{t} ; \alpha, \lambda_{+}, \lambda_{-}\right)\right)
\end{array}\right.
$$

Then there is a measure $\mathbf{Q}_{t}$ equivalent to $\mathbf{P}_{t}$ such that $\varepsilon_{t}+k_{t} \sim \operatorname{stdCTS}\left(\alpha, \tilde{\lambda}_{+}(t), \tilde{\lambda}_{-}(t)\right)$ on the measure $\mathbf{Q}_{t}$ where

$$
k_{t}=\lambda_{t}+\frac{1}{\sigma_{t}}\left(L_{C T S}\left(\sigma_{t} ; \alpha, \tilde{\lambda}_{+}(t), \tilde{\lambda}_{-}(t)\right)-L_{C T S}\left(\sigma_{t} ; \alpha, \lambda_{+}, \lambda_{-}\right)\right) .
$$

The stock price dynamic, deduced from Proposition 6.1 is

$$
\left\{\begin{array}{l}
\log \left(\frac{S_{t}}{S_{t-1}}\right)=r_{t}-d_{t}-L_{C T S}\left(\sigma_{t} ; \alpha, \tilde{\lambda}_{+}(t), \tilde{\lambda}_{-}(t)\right)+\sigma_{t} \xi_{t} \\
\xi_{t} \stackrel{\mathrm{d}}{=} \varepsilon_{t}+k_{t} \sim \operatorname{stdCTS}\left(\alpha, \tilde{\lambda}_{+}(t), \tilde{\lambda}_{-}(t)\right)
\end{array}, t \in \mathbb{N}\right.
$$

having the following variance process

$$
\sigma_{t}^{2}=\left(\alpha_{0}+\alpha_{1} \sigma_{t-1}^{2}\left(\xi_{t-1}-k_{t-1}\right)^{2}+\beta_{1} \sigma_{t-1}^{2}\right) \wedge \rho
$$

The stock price dynamic is called the the CTS-GARCH option pricing model, where $\tilde{\lambda}_{+}(t)$ and $\tilde{\lambda}_{-}(t)$ satisfy condition (27), and $k_{t}$ is equal to equation (28). Under the CTS-GARCH option pricing model, the stock price $S_{t}$ at time $t>0$ is given by

$$
S_{t}=S_{0} \exp \left(\sum_{j=1}^{t}\left(r_{j}-d_{j}-L_{C T S}\left(\sigma_{j} ; \alpha, \tilde{\lambda}_{+}(t), \tilde{\lambda}_{-}(t)\right)+\sigma_{j} \xi_{j}\right)\right),
$$


and $E\left[S_{t} \mid \mathcal{F}_{t-1}\right]=S_{t-1} e^{r_{t}-d_{t}}$

\subsection{MTS-GARCH Model}

The tempered stable GARCH model with the sequence $\left(\varepsilon_{t}\right)_{t \in \mathbb{N}}$ of iid random variables is called the MTS-GARCH model, if $\varepsilon_{t} \sim \operatorname{stdMTS}\left(\alpha, \lambda_{+}, \lambda_{-}\right)$for all $t \in \mathbb{N}$. Since $E\left[e^{x \varepsilon_{t}}\right]<\infty$ if $x \in\left(-\lambda_{-}, \lambda_{+}\right), \rho$ has to be in the interval $\left(0, \lambda_{+}^{2}\right)$. By Corollary 3.5 (b), we can prove the following proposition.

Proposition 6.2. Consider the MTS-GARCH model. Let $T \in \mathbb{N}$ be a time horizon, fix natural number $t \leq T$. Suppose $\tilde{\lambda}_{+}(t)$ and $\tilde{\lambda}_{-}(t)$ satisfy the following conditions:

$$
\left(\begin{array}{l}
\tilde{\lambda}_{+}(t)^{2}>\rho \\
\tilde{\lambda}_{+}(t)^{\alpha-2}+\tilde{\lambda}_{-}(t)^{\alpha-2}=\lambda_{+}^{\alpha-2}+\lambda_{-}^{\alpha-2} \\
\frac{\Gamma\left(\frac{1-\alpha}{2}\right)\left(\lambda_{+}^{\alpha-1}-\lambda_{-}^{\alpha-1}-\tilde{\lambda}_{+}(t)^{\alpha-1}+\tilde{\lambda}_{-}(t)^{\alpha-1}\right)}{\sqrt{\pi} \Gamma\left(1-\frac{\alpha}{2}\right)\left(\lambda_{+}^{\alpha-2}+\lambda_{-}^{\alpha-2}\right)} \\
\quad=\lambda_{t}+\frac{1}{\sigma_{t}}\left(L_{M T S}\left(\sigma_{t} ; \alpha, \tilde{\lambda}_{+}(t), \tilde{\lambda}_{-}(t)\right)-L_{M T S}\left(\sigma_{t} ; \alpha, \lambda_{+}, \lambda_{-}\right)\right)
\end{array}\right.
$$

Then there is a measure $\mathbf{Q}_{t}$ equivalent to $\mathbf{P}_{t}$ such that $\varepsilon_{t}+k_{t} \sim \operatorname{stdMTS}\left(\alpha, \tilde{\lambda}_{+}(t), \tilde{\lambda}_{-}(t)\right)$ on the measure $\mathbf{Q}_{t}$ where

$$
k_{t}=\lambda_{t}+\frac{1}{\sigma_{t}}\left(L_{M T S}\left(\sigma_{t} ; \alpha, \tilde{\lambda}_{+}(t), \tilde{\lambda}_{-}(t)\right)-L_{M T S}\left(\sigma_{t} ; \alpha, \lambda_{+}, \lambda_{-}\right)\right) .
$$

The stock price dynamic, deduced from Proposition 6.2 is

$$
\left\{\begin{array}{l}
\log \left(\frac{S_{t}}{S_{t-1}}\right)=r_{t}-d_{t}-L_{M T S}\left(\sigma_{t} ; \alpha, \tilde{\lambda}_{+}(t), \tilde{\lambda}_{-}(t)\right)+\sigma_{t} \xi_{t} \\
\xi_{t} \stackrel{\mathrm{d}}{=} \varepsilon_{t}+k_{t} \sim \operatorname{stdMTS}\left(\alpha, \tilde{\lambda}_{+}(t), \tilde{\lambda}_{-}(t)\right)
\end{array}, t \in \mathbb{N}\right.
$$

having the following variance process

$$
\sigma_{t}^{2}=\left(\alpha_{0}+\alpha_{1} \sigma_{t-1}^{2}\left(\xi_{t-1}-k_{t-1}\right)^{2}+\beta_{1} \sigma_{t-1}^{2}\right) \wedge \rho
$$

The stock price dynamic is called the the MTS-GARCH option pricing model, where $\tilde{\lambda}_{+}(t)$ and $\tilde{\lambda}_{-}(t)$ satisfy condition (31), and $k_{t}$ is equal to equation (32). Under the MTS-GARCH option pricing model, the stock price $S_{t}$ at time $t>0$ is given by

$$
S_{t}=S_{0} \exp \left(\sum_{j=1}^{t}\left(r_{j}-d_{j}-L_{M T S}\left(\sigma_{j} ; \alpha, \tilde{\lambda}_{+}(t), \tilde{\lambda}_{-}(t)\right)+\sigma_{j} \xi_{j}\right)\right)
$$

and $E\left[S_{t} \mid \mathcal{F}_{t-1}\right]=S_{t-1} e^{r_{t}-d_{t}}$ 


\subsection{Generating Algorithm for the CTS-GARCH and MTS-GARCH Option Pricing Models}

Assume that the GARCH parameters $\left(\alpha_{0}, \alpha_{1}\right.$, and $\left.\beta_{1}\right)$ the standard CTS [MTS] parameters $\left(\alpha, \lambda_{+}\right.$, and $\left.\lambda_{-}\right)$the constant market price of risk $\lambda_{t}=\lambda$, and the conditional variance $\sigma_{t_{0}}^{2}$ of the initial time $t_{0}$ are estimated from the historical data. Then we can generate the risk-neutral process for the CTS-GARCH option pricing model by the following algorithm.

\section{Algorithm:}

1. Initialize $t:=t_{0}$.

2. Find the parameters $\tilde{\lambda}_{+}(t)$ and $\tilde{\lambda}_{-}(t)$ satisfying condition $(27)$.

3. Generate random number $\xi_{t} \sim \operatorname{stdCTS}\left(\alpha, \tilde{\lambda}_{+}(t), \tilde{\lambda}_{-}(t)\right)$.

4. Let $\log \left(\frac{S_{t}}{S_{t-1}}\right)$ be equal to equation $(29)$.

5. Let $k_{t}$ be equal to equation (28).

6. Set $t=t+1$ and then substitute $\sigma_{t}^{2}=\left(\alpha_{0}+\alpha_{1} \sigma_{t-1}^{2}\left(\xi_{t-1}-k_{t-1}\right)^{2}+\beta_{1} \sigma_{t-1}^{2}\right) \wedge \rho$.

7. Repeat $2 \sim 6$ until $t>T$.

We can generate the risk-neutral process for the MTS-GARCH option pricing model by changing the above algorithm as following:

$2^{\prime}$. Find the parameters $\tilde{\lambda}_{+}(t)$ and $\tilde{\lambda}_{-}(t)$ satisfying condition $(31)$.

$3^{\prime}$. Generate random number $\xi_{t} \sim \operatorname{stdMTS}\left(\alpha, \tilde{\lambda}_{+}(t), \tilde{\lambda}_{-}(t)\right)$.

$4^{\prime}$. Let $\log \left(\frac{S_{t}}{S_{t-1}}\right)$ be equal to equation $(33)$.

$5^{\prime}$. Let $k_{t}$ be equal to equation (32).

\subsection{KR-GARCH Model}

The tempered stable GARCH model with the sequence $\left(\varepsilon_{t}\right)_{t \in \mathbb{N}}$ of iid random variables is called the $K R-G A R C H$ model, if $\varepsilon_{t} \sim \operatorname{stdKR}\left(\alpha, r_{+}, r_{-}, p_{+}, p_{-}\right)$for all $t \in \mathbb{N}$ and the parameters $p_{+}$and $p_{-}$satisfy condition (23). Since $E\left[e^{x \varepsilon t}\right]<\infty$ if $x \in\left(-1 / r_{-}, 1 / r_{+}\right), \rho$ must be in the interval $\left(0,1 / r_{+}^{2}\right)$. 
Proposition 6.3. Consider the KR-GARCH model. Let $T \in \mathbb{N}$ be a time horizon, fix natural number $t \leq T$. Suppose $\tilde{r}_{+}(t), \tilde{r}_{-}(t), \tilde{p}_{+}(t)$, and $\tilde{p}_{-}(t)$ satisfy the following conditions:

$$
\left(\begin{array}{l}
\tilde{r}_{+}(t)^{-2}>\rho \\
\frac{\tilde{p}_{+}(t)+2}{\tilde{p}_{+}(t)+\alpha} \tilde{r}_{+}(t)^{\alpha-2}=\frac{p_{+}+2}{p_{+}+\alpha} r_{+}^{\alpha-2} \\
\frac{\tilde{p}_{-}(t)+2}{\tilde{p}_{-}(t)+\alpha} \tilde{r}_{-}(t)^{\alpha-2}=\frac{p_{-}+2}{p_{-}+\alpha} r_{-}^{\alpha-2} \\
\frac{\Gamma(1-\alpha)}{2 \Gamma(2-\alpha)}\left(\frac{\tilde{p}_{+}(t)+2}{\tilde{r}_{+}(t)\left(\tilde{p}_{+}(t)+1\right)}-\frac{\tilde{p}_{-}(t)+2}{\tilde{r}_{-}(t)\left(\tilde{p}_{-}(t)+1\right)}-\frac{p_{+}+2}{r_{+}\left(p_{+}+1\right)}+\frac{p_{-}+2}{r_{-}\left(p_{-}+1\right)}\right) \\
=\lambda_{t}+\frac{1}{\sigma_{t}}\left(L_{K R}\left(\sigma_{t} ; \alpha, \tilde{r}_{+}(t), \tilde{r}_{-}(t), \tilde{p}_{+}(t), \tilde{p}_{-}(t)\right)-L_{K R}\left(\sigma_{t} ; \alpha, r_{+}, r_{-}, p_{+}, p_{-}\right)\right)
\end{array}\right)
$$

Then there is a measure $\mathbf{Q}_{t}$ equivalent to $\mathbf{P}_{t}$ such that $\varepsilon_{t}+k_{t} \sim \operatorname{stdKR}\left(\alpha, \tilde{r}_{+}(t), \tilde{r}_{-}(t), \tilde{p}_{+}(t)\right.$, $\left.\tilde{p}_{-}(t)\right)$ on the measure $\mathbf{Q}_{t}$ where

$$
k_{t}=\lambda_{t}+\frac{1}{\sigma_{t}}\left(L_{K R}\left(\sigma_{t} ; \alpha, \tilde{r}_{+}(t), \tilde{r}_{-}(t), \tilde{p}_{+}(t), \tilde{p}_{-}(t)\right)-L_{K R}\left(\sigma_{t} ; \alpha, r_{+}, r_{-}, p_{+}, p_{-}\right)\right) .
$$

The stock price dynamic, deduced from Proposition 6.3 is

$$
\left(\begin{array}{l}
\log \left(\frac{S_{t}}{S_{t-1}}\right)=r_{t}-d_{t}-L_{K R}\left(\sigma_{t} ; \alpha, \tilde{r}_{+}(t), \tilde{r}_{-}(t), \tilde{p}_{+}(t), \tilde{p}_{-}(t)\right)+\sigma_{t} \xi_{t} \\
\xi_{t} \stackrel{\stackrel{\mathrm{d}}{=}}{=} \varepsilon_{t}+k_{t} \sim \operatorname{stdKR}\left(\alpha, \tilde{r}_{+}(t), \tilde{r}_{-}(t), \tilde{p}_{+}(t), \tilde{p}_{-}(t)\right)
\end{array}, t \in \mathbb{N}\right.
$$

possessing the following variance process

$$
\sigma_{t}^{2}=\left(\alpha_{0}+\alpha_{1} \sigma_{t-1}^{2}\left(\xi_{t-1}-k_{t-1}\right)^{2}+\beta_{1} \sigma_{t-1}^{2}\right) \wedge \rho
$$

is called the the KR-GARCH option pricing model, where $\tilde{r}_{+}(t), \tilde{r}_{-}(t), \tilde{p}_{+}(t)$, and $\tilde{p}_{-}(t)$ satisfy the condition (35), and $k_{t}$ is equal to the equation (36). Under the MTS-GARCH option pricing model, the stock price $S_{t}$ at time $t>0$ is given by

$$
S_{t}=S_{0} \exp \left(\sum_{j=1}^{t}\left(r_{j}-d_{j}-L_{K R}\left(\sigma_{t} ; \alpha, \tilde{r}_{+}(t), \tilde{r}_{-}(t), \tilde{p}_{+}(t), \tilde{p}_{-}(t)\right)+\sigma_{j} \xi_{j}\right)\right)
$$

and $E\left[S_{t} \mid \mathcal{F}_{t-1}\right]=S_{t-1} e^{r_{t}-d_{t}}$.

Assume that the GARCH parameters $\left(\alpha_{0}, \alpha_{1}\right.$, and $\left.\beta_{1}\right)$, the standard MTS parameters $(\alpha$, $r_{+}, r_{-}, p_{+}$, and $\left.p_{-}\right)$, the constant market price of risk $\lambda_{t}=\lambda$, and the conditional variance $\sigma_{t_{0}}^{2}$ of the initial time $t_{0}$ are estimated from historical data. Then we can generate the tempered stable GARCH option pricing model based on the standard KR distribution by the following algorithm.

\section{Algorithm:}

1. Initialize $t:=t_{0}$. 
2. Find the parameters $\tilde{r}_{+}(t), \tilde{r}_{-}(t), \tilde{p}_{+}(t)$ and $\tilde{p}_{-}(t)$ satisfying the conditions in $(35)$.

3. Generate random number $\xi_{t} \sim \operatorname{stdKR}\left(\alpha, \tilde{r}_{+}(t), \tilde{r}_{-}(t), \tilde{p}_{+}(t), \tilde{p}_{-}(t)\right)$.

4. Put $\log \left(\frac{S_{t}}{S_{t-1}}\right)=r_{t}-d_{t}-L_{K R}\left(\alpha, \tilde{r}_{+}(t), \tilde{r}_{-}(t), \tilde{p}_{+}(t), \tilde{p}_{-}(t)\right)+\sigma_{t} \xi_{t}$

5. Let $k_{t}=\lambda+\frac{1}{\sigma_{t}}\left(L_{K R}\left(\alpha, \tilde{r}_{+}(t), \tilde{r}_{-}(t), \tilde{p}_{+}(t), \tilde{p}_{-}(t)\right)-L_{K R}\left(\alpha, r_{+}, \tilde{r}_{-}, \tilde{p}_{+}, \tilde{p}_{-}\right)\right)$

6. Set $t=t+1$ and then substitute $\sigma_{t}^{2}=\left(\alpha_{0}+\alpha_{1} \sigma_{t-1}^{2}\left(\xi_{t-1}-k_{t-1}\right)^{2}+\beta_{1} \sigma_{t-1}^{2}\right) \wedge \rho$.

7. Repeat $2 \sim 6$ until $t>T$.

In Step 2 in this algorithm, we have to find the solution with four parameters satisfying the condition in (35). The solution is not unique. There are many way to select one of them. One way is to select one solution which minimizes the square root error between the market option prices and the simulated option prices. Another way is by fixing the parameter $\tilde{r}_{+}(t)=r_{+}$. Then, since $\rho<r_{+}$, the first condition in (35) is naturally satisfied.

\section{Empirical for the tempered stable GARCH models}

\subsection{Estimation of the Parameters for the GARCH models}

In this section we report on the maximum likelihood estimation (MLE) of the normalGARCH and MTS-GARCH models. In our empirical study, we use a sample that includes the S\&P 500 index (SPX) and the following three stocks: Intel (INTC), Microsoft (MSFT), and Amazon.com (AMZN). The time series of the prices of $\mathrm{AMZN}^{1}$ is from March 11, 2001 to March 10, 2006, and the others are from March 11, 1996 to March 10, 2006. For the daily risk-free rate, we select the appropriate zero-coupon rate supplied by IVYDB and convert it to a continuous compound rate. To simplify the estimation, we impose a constant market price of risk $\lambda$. For INTC, MSFT, and AMZN we use the adjusted-closing prices to estimate the market parameters with the MLE. The adjusted-closing prices adjust for all applicable stock splits and stock dividend distributions. For this reason, we modify the stock price dynamic of INTC, MSFT, and AMZN as follows

$$
\log \left(\frac{\hat{S}_{t}}{\hat{S}_{t-1}}\right)=r_{t}+\lambda_{t} \sigma_{t}-L\left(\sigma_{t}\right)+\sigma_{t} \varepsilon_{t}, t \in \mathbb{N},
$$

\footnotetext{
${ }^{1}$ Amazon.com was listed on 1997. Price movement after listing was turbulent. In order to obtain a more typical time series for this stock, we selected March 2001 as the initial date.
} 
where $\hat{S}_{t}$ is the adjusted-closing prices. The historical prices of the SPX provided by IVYDB are not adjusted-closing prices but just closing prices. Consequently, instead of the adjusted price, IVYDB provides daily data for continuous dividends. Hence, we can use the dynamic defined by (25) without modification for estimating the market parameters of the SPX.

Our estimation procedure is as follows. First, we estimate the parameters $\alpha_{0}, \alpha_{1}, \beta_{1}$, and the constant market price of risk $\lambda$ from the normal-GARCH model. Second, we fix $\alpha_{0}, \alpha_{1}, \beta_{1}$, and $\lambda$ and then estimate the parameters of those three standard tempered stable innovation distributions: the standard CTS, the standard MTS, and the standard KR distributions. Here we assume that $\sigma_{0}^{2}=\alpha_{0} /\left(1-\alpha_{1}-\beta_{1}\right)$ and $\rho=\max \left\{\sigma_{t}^{2}: t\right.$ is the observed date $\}$. We list the estimated GARCH parameters in Table 3, and the parameters for the three standard tempered stable distributions in Table 4.

For the assessment of the goodness-of-fit, we use the Kolmogrov-Smirnov (KS) test. We also calculate the Anderson-Darling (AD) statistic to better evaluate the tail fit. We define the null hypotheses as follows :

$H_{0}(\mathrm{GARCH})$ : The residuals follow the standard normal distribution.

$H_{0}(\mathrm{CTS}-\mathrm{GARCH})$ : The residuals follow the standard CTS distribution.

$H_{0}$ (MTS-GARCH): The residuals follow the standard MTS distribution.

$H_{0}(\mathrm{KR}-\mathrm{GARCH})$ : The residuals follow the standard KR distribution.

Table 5 provides the KS statistic with their $p$-values. Based on the results reported in the table, we can assert that

1. $H_{0}(\mathrm{GARCH})$ is rejected for all tested datasets.

2. $H_{0}(\mathrm{CTS}-\mathrm{GARCH})$ is not rejected at the $7 \%$ significance level for SPX, $15 \%$ significance level for INTC, 3\% significance level for MSFT, and 90\% significance level for AMZN.

3. $H_{0}(\mathrm{MTS}-\mathrm{GARCH})$ is not rejected at the $6 \%$ significance level for SPX, $15 \%$ significance level for INTC, $3 \%$ significance level for MSFT, and 2\% significance level for AMZN.

4. $H_{0}(\mathrm{KR}-\mathrm{GARCH})$ is not rejected at the $10 \%$ significance level for $\mathrm{SPX}, 40 \%$ significance level for INTC, 60\% significance level for MSFT, and 90\% significance level for AMZN. 
Moreover, we can see that the values of the AD statistic for the three standard tempered stable cases are significantly smaller than that for the standard normal case. That means the tempered stable GARCH models explain the extreme event of the real innovation process better than the normal-GARCH model does.

\subsection{The Out-of-sample Forecasting Performance}

In this section, we discuss the out-of-sample forecasting performance of the three subclasses of the tempered stable GARCH models (the CTS-GARCH, the MTS-GARCH, and the KR-GARCH models) for the S\&P 500 call option prices. We then compare the performance of the tempered stable GARCH models with the performance of the Black-Scholes (BS) model by historical volatility, and of the CGMY model with the Esscher transform.

The arbitrage free price of a call option on time $t<T$ with strike $K$ and maturity $T$ is given by

$$
C_{t}=\exp \left(-\sum_{j=t+1}^{T} r_{j}\right) E\left[\left(S_{T}-K\right)^{+} \mid \mathfrak{F}_{t}\right],
$$

Since we do not have an efficient analytical form for the option price (38) for the CTSGARCH, the MTS-GARCH, or the KR-GARCH option pricing models, the call option prices are determined by the quasi-Monte Carlo (QMC) method. For the calculation option prices of the CGMY model with Esscher transform, we find $\theta$ satisfying (5), and then followed the Fourier transform method of Carr and Madan (1999) and Lewis (2001).

We take a set of closing prices on March 10, 2006 expiring on March 17, 2006. The number of trading days to the option's expiration is five. The risk-neutral interest rate and dividend rate on March 10, 2006 are $r=0.0463$ and $d=0.0194$, respectively. We estimate the market parameters for the historical daily data of the S\&P 500 index from March 11, 1996 to March 10, 2006. The historical volatility for the historical data is 0.2205 . The market parameter for the CGMY model estimation gives the following market parameters: $\alpha=0.9147, C=2.7947, \lambda_{+}=79.2511, \lambda_{-}=72.8717$, and $\mu=0.1250$. The KS statistic and its $p$-value of the estimation for the CGMY model are 0.0097 and 0.9732 , respectively. The parameter $\theta$ of the Esscher transform satisfying (5) is equal to $\theta=8.4920$. The market parameters of the three tempered stable GARCH models for the SPX price which is the underlying for the SPX call option were given in the previous section. With those market 
parameters, the risk-neutral return processes of the CTS-GARCH and the MTS-GARCH models are generated by the algorithm in Section 6.3, and the risk-neutral return processes of the KR-GARCH models are generated by the algorithm in Section 6.4. We use the inverse transform algorithm for generate the standard CTS, the standard MTS, and the standard KR distributed random numbers. We need the cumulative density function and uniformly distributed random numbers to generate random number using the inverse transform algorithm. We compute the values of the cumulative density functions by numerical method. Uniformly distributed random number is obtained by the Sobol' method (Sobol (1967)). According to the numerical example of the QMC method for Black-Scholes European call option pricing presented in Glasserman (2003), the convergence rate of the QMC method using the Sobol' points with five time steps is close to $\mathcal{O}(1 / n)$, while the convergence rate of Monte Carlo method is $\mathcal{O}(1 / \sqrt{n})$, where $n$ is the number of paths. For GARCH models given in this section, we cannot determine the exact convergence rate, but we can say that the QMC method can have a better performance rate than the Monte Carlo method. For that reason, we simulate 4096 sample paths for calculating the option prices for each model.

For the KR-GARCH model, we fix the parameter $\tilde{r}_{+}(t)=r_{+}$in this test. There are several reasonable ways to choose the initial variance $\sigma_{0}^{2}$, which strongly influences the model behavior. One possibility is to take the last value in the estimated series or alternatively to choose $\sigma_{0}^{2}=\alpha_{0} /\left(1-\alpha_{1}-\beta_{1}\right)$ which is a fraction of the stationary variance. Here, we choose the first method.

To measure the performance of the three models, we will use AAE, APE, and RMSE. The values of the errors for each model are presented in Table 6, and market call prices and call prices of the BS model, the CGMY model with Esscher transform, and three tempered stable models are drawn in Figure 2. In addition, the implied volatility curves for each price is described in Figure 3. This figure shows the implied volatility curves for the bid and offer prices in order to see the difference between the market and the model volatilities.

The errors for three tempered stable GARCH models are smaller than the errors for the BS, CGMY, and the normal-GARCH models. In particular, the value of the errors for the MTS-GARCH model are smaller than the errors for any other models tested. The implied volatility curves for the three tempered stable GARCH models are more similar 
to the implied volatility for the bid and offer prices than the curves for the CGMY and normal-GARCH models. Also, the implied volatility curves for the CTS-GARCH and the KR-GARCH model are more convex than the curve for the MTS-GARCH model. Hence, the curves for the CTS-GARCH and the KR-GARCH models seem more realistic than the curve for the CGMY and normal-GARCH models as well a for the curve for the MTS-GARCH model. The performance of the KR-GARCH model is not better than the CTS-GARCH nor the MTS-GARCH models, but the KR-GARCH model has one more degree of freedom. We fix the parameter $\tilde{r}_{+}(t)=r_{+}$in this test. If we select parameters under which minimize the square root error between the market call prices and the simulated call prices, then we could obtain superior performance, and calibrate the risk neutral measure for the current call price.

\section{Conclusion}

In this paper, we investigate two types of market models: the continuous market model and the discrete market model. The former model presented in this paper is the extended tempered stable market model that we have named the KR model. This model is an extension of the CGMY model that allows for more flexible parameters than both the CGMY model and the MTS model. This greater flexibility allows for a more efficient means for calibrating the risk-neutral measure. With respect to the discrete market model, we present a new nonnormal GARCH model with infinitely divisible distributed innovation. More specifically, we consider three subclasses of the GARCH model: the CTS-GARCH model, MTS-GARCH model, and the KR-GARCH model. Not only do these models describe volatility clustering, but other tail characteristics that have been observed for asset returns. Hence, these models are more realistic GARCH models that can be employed in option pricing.

The three tempered stable GARCH models investigated in this paper are not rejected in statistical hypothesis testing, while the normal-GARCH model is rejected. The reason

for the good statistical results for tempered stable GARCH models relative to the normalGARCH model is that skewness and fat-tail property of their innovation are taken into account. Moreover, the tempered stable GARCH models have better forecasting performance than the normal-GARCH model and the CGMY model with Esser transform, in addition to generating a more realistic implied volatility curve. Consequently, the clustering volatility 
with the tempered stable innovation explains the behavior of stock and option markets better than the Markovian model of the exponential CGMY distribution and the clustering volatility with the standard normal residual distribution.

\section{References}

[1] Black, F., Scholes, M., 1973. The Pricing of Options and Corporate Liabilities.The Journal of Political Economy 81, 3, 637-654.

[2] Boyarchenko, S. I., Levendorskii, S. Z., 2000. Option pricing for truncated Lévyprocesses.International Journal of Theoretical and Applied Finance 3, 3, 549-552.

[3] Carr, P., Geman, H., Madan, D., Yor, M., 2002. The Fine Structure of Asset Returns: An Empirical Investigation.Journal of Business 75, 2, 305-332.

[4] Carr, P., Madan, D., 1999. Option Valuation Using the Fast Fourier Transform.The Journal of Computational Finance 2, 4, 61-73.

[5] Cont, R., Tankov, P., 2004. Financial Modelling with Jump Processes.Chapman \& Hall / CRC.

[6] Duan, J.-C., 1995. The GARCH Option Pricing Model.Mathematical Finance 5, 1, 13-32.

[7] Gerber, H.U., Shiu, E.S.W., 1994. Option pricing by Esscher transforms (with disscusions).Transactions of the Society of Actuaries 46, 99-191.

[8] Gerber, H.U., Shiu, E.S.W., 1996. Actuarial bridges to dynamic hedging and option pricing.Insurance : Mathmatics and Economics, 18, 3, 183-218.

[9] Glasserman, P., 2003. Monte Carlo Methods in Financial Engineering.Springer.

[10] Kim, Y. S., 2005. The Modified Tempered Stable Processes with Application to Finance.Ph.D thesis, Sogang University.

[11] Kim, Y. S., Lee, J. H., 2006. The Relative Entropy in CGMY Processes and Its Applications to Finance.To appear inMathematical Methods of Operations Research.

[12] Kim, Y. S., Rachev, S. T., Bianchi, M. L., Fabozzi, F. J., 2007. A New Tempered Stable Distribution and Its Application to Finance. forthcoming in Georg Bol, Svetlozar T. Rachev, and Reinold Wuerth (Eds.), Risk Assessment: Decisions in Banking and Finance, Physika Verlag, Springer 2007.

[13] Kim, Y. S., Rachev, S. T., Chung, D. M., 2006. The Modified Tempered Stable Distribution, GARCHModels and Option Pricing.Technical report, Chair of Econometrics, Statistics and Mathematical Finance School of Economics and Business Engineering University of Karlsruhe (http://www.statistik.unikarlsruhe.de/download/KimRachevChung.pdf).

[14] Koponen, I., 1995. Analytic approach to the problem of convergence of truncated Lévy flights towards the Gaussian stochastic process.Physical Review E, 52, 1197-1199.

[15] Lewis, A.L., 2001. A Simple Option Formula for General Jump-Diffusion and Other Exponential Lévy Processes.Available at http://optioncity.net/pubs/ExpLevy.pdf

[16] Mandelbrot, B. B., 1963. New Methods in Statistical Economics.Journal of Political Economy, 71, 421-440.

[17] Fujiwara, T., Miyahara, Y., 2003. The Minimal Entropy Martingale Measures for Geometric Lévy Processes.Finance \& Stochastics 7, 509-531.

[18] Miyahara, Y., 2004. The [GLP \& MEMM] Pricing Model and Its Calibration Problems.Discussion Papers in Economics, Nagoya City University 397, 1-30. 
[19] Menn, C., Rachev, S. T., 2005a. A GARCH Option Pricing Model with $\alpha$-stable Innovations.European Journal of Operational Research, 163, 201-209.

[20] Menn, C., Rachev, S. T., 2005b. Smoothly Truncated Stable Distributions, GARCH-Models, and Option Pricing.Technical report, Chair of Econometrics, Statistics and Mathematical Finance School of Economics and Business Engineering University of Karlsruhe (http://www.statistik.unikarlsruhe.de/download/tr_smoothly_truncated.pdf).

[21] Rachev, S. T., Menn, C., Fabozzi, F. J., 2005. Fat-Tailed and Skewed Asset Return Distributions: Implications for Risk Management, Portfolio selection, and Option Pricing.John Wiley \& Sons.

[22] Rachev, S. T., Mitnik, S., 2000. Stable Paretian Models in Finance.John Wiley \& Sons.

[23] Rosiński, J., 2006. Tempering Stable Processes.(http://www.math.utk.edu/ rosinski/Manuscripts /tstableF.pdf), To appear in the Stochastic Processes and Their Applications.

[24] Sato, K., 1999. Lévy Processes and Infinitely Divisible Distributions.Cambridge University Press.

[25] Schoutens, W., 2003. Lévy Processes in Finance: Pricing Financial Derivatives.John Wiley \& Sons.

[26] Sobol', I.M., 1967. On the distribution of points in a cube and the approximate evaluation of integrals.USSR Journal of Computational Mathematics and Mathematical Physics, (English translation), 7, 784-802. 
Table 1: Market Parameters for the S\&P 500 Index

\begin{tabular}{|c|c|c|c|c|c|c|c|c|c|}
\hline Date & Model & & & & Parameters & & & & $K S(p$-value $)$ \\
\hline June 8,2000 & KR & $\mu$ & $\alpha$ & $k_{+}$ & $k_{-}$ & $r_{+}$ & $r_{-}$ & $p_{+}=p_{-}$ & \\
\hline -June 8, 2005 & & 0.1106 & 0.9106 & 3508.6026 & 3161.1567 & 0.0126 & 0.0142 & 20.4773 & $0.0094(0.9845)$ \\
\hline & CGMY & $\mu$ & $\alpha$ & C & $\lambda_{+}$ & $\lambda_{-}$ & & & \\
\hline & & 0.1106 & 0.9146 & 2.9742 & 73.3250 & 82.1808 & & & $0.0097(0.9784)$ \\
\hline & MTS & $\mu$ & $\alpha$ & $C$ & $\lambda_{+}$ & $\lambda_{-}$ & & & \\
\hline & & 0.1107 & 0.9058 & 2.5974 & 106.5000 & 95.0007 & & & $0.0098(0.9766)$ \\
\hline July 13,2000 & KR & $\begin{array}{c}\mu \\
\mu-1135\end{array}$ & $\begin{array}{c}\alpha \\
0-8779\end{array}$ & $\begin{array}{c}k+ \\
k 582\end{array}$ & $k_{-}$ & $r_{+}$ & $r_{-}$ & $p_{+}=p_{-}$ & $00091(0.9882)$ \\
\hline & CGMY & $\mu$ & $\alpha$ & $\begin{array}{c}3082.4302 \\
C\end{array}$ & $\begin{array}{c}3223.1218 \\
\lambda_{+}\end{array}$ & $\begin{array}{c}0.0122 \\
\lambda_{-}\end{array}$ & & & $0.0091(0.9882)$ \\
\hline & & 0.1135 & 0.8990 & 3.2190 & 74.0199 & 83.2370 & & & $0.0095(0.9815)$ \\
\hline & MTS & $\mu$ & $\alpha$ & C & $\lambda_{+}$ & $\lambda_{-}$ & & & \\
\hline & & 0.1135 & 0.8954 & 2.7249 & 107.0000 & 94.8782 & & & $0.0096(0.9800)$ \\
\hline August 10,2000 & KR & $\mu$ & $\alpha$ & $k_{+}$ & $k_{-}$ & $r_{+}$ & $r_{-}$ & $p_{+}=p_{-}$ & \\
\hline -August 10,2005 & & 0.1133 & 0.8608 & 3472.2100 & 3118.2710 & 0.0120 & 0.0136 & 18.3149 & $0.0089(0.9906)$ \\
\hline & CGMY & $\mu$ & $\alpha$ & C & $\lambda_{+}$ & $\lambda_{-}$ & & & \\
\hline & & 0.1133 & 0.9072 & 3.0370 & 73.1453 & 82.3510 & & & $0.0094(0.9822)$ \\
\hline & MTS & $\mu$ & $\alpha$ & $C$ & $\lambda_{+}$ & $\lambda_{-}$ & & & \\
\hline September 14,2000 & KR. & $\frac{0.1134}{\mu}$ & $\frac{0.8815}{\alpha}$ & $\frac{2.9015}{k+}$ & $\frac{106.0000}{k}$ & $\frac{94.8266}{r+}$ & & & $0.0094(0.9838)$ \\
\hline -September 14, 2005 & ח & 0.1120 & 0.9749 & 3377.4398 & $\begin{array}{l}\kappa- \\
3040.6725\end{array}$ & $\begin{array}{c}r+ \\
0.0141\end{array}$ & 0.0161 & $\begin{array}{c}p_{+}=p- \\
26.5375\end{array}$ & $0.0092(0.9857)$ \\
\hline & CGMY & $\mu$ & $\alpha$ & $C$ & $\lambda_{+}$ & $\lambda_{-}$ & & & \\
\hline & & 0.1120 & 0.9131 & 2.9046 & 72.6579 & 81.5166 & & & $0.0093(0.9845)$ \\
\hline & MTS & $\mu$ & $\alpha$ & $C$ & $\lambda_{+}$ & $\lambda_{-}$ & & & \\
\hline & & 0.1120 & 0.9043 & 2.5346 & 105.5000 & 94.0566 & & & $0.0093(0.9837)$ \\
\hline October 12, 2000 & KR & $\mu$ & $\alpha$ & $k_{+}$ & $k_{-}$ & $r_{+}$ & $r_{-}$ & $p_{+}=p_{-}$ & \\
\hline -October 12, 2005 & CGMY & $\begin{array}{c}0.1044 \\
\mu\end{array}$ & $\begin{array}{c}0.9040 \\
\alpha\end{array}$ & $\begin{array}{c}3302.0528 \\
C\end{array}$ & $\begin{array}{c}2984.3026 \\
\lambda+\end{array}$ & 0.0126 & 0.0142 & 19.8555 & $0.0089(0.9897)$ \\
\hline & & 0.1044 & 0.9191 & 2.7870 & 72.3754 & 80.8017 & & & $0.0093(0.9838)$ \\
\hline & MTS & $\mu$ & $\alpha$ & C & $\lambda_{+}$ & $\lambda_{-}$ & & & \\
\hline & & 0.1044 & 0.8935 & 2.6605 & 104.5000 & 93.9012 & & & $0.0091(0.9867)$ \\
\hline November 9,2000 & $\mathrm{KR}$ & $\mu$ & $\alpha$ & $k_{+}$ & $k_{-}$ & $r_{+}$ & $r_{-}$ & $p_{+}=p_{-}$ & \\
\hline -November 9, 2005 & & 0.1093 & 0.9050 & 3322.8650 & 3007.4244 & 0.0126 & 0.0142 & 19.9789 & $0.0090(0.9885)$ \\
\hline & CGMY & $\mu$ & $\alpha$ & C & $\lambda_{+}$ & $\lambda_{-}$ & & & \\
\hline & & 0.1093 & 0.9181 & 2.8050 & 72.8032 & 81.1674 & & & $0.0093(0.9827)$ \\
\hline & MTS & $\mu$ & $\begin{array}{c}\alpha \\
09940\end{array}$ & $\begin{array}{c}C \\
26522\end{array}$ & $\stackrel{\lambda_{+}}{ }$ & $\lambda_{-}{ }_{9433}$ & & & $00092(0.9850)$ \\
\hline December 14,2000 & KR & $\mu$ & $\frac{0.050}{\alpha}$ & $k_{+}$ & $k_{-}$ & $r_{+}$ & $r$ & $p_{+}=p_{-}$ & $0.0092(0.9050)$ \\
\hline -December 14,2005 & & 0.1149 & 0.8364 & 3416.2155 & 3086.5717 & 0.0118 & 0.0133 & 17.5284 & $0.0086(0.9924)$ \\
\hline & CGMY & $\mu$ & $\alpha$ & C & $\lambda_{+}$ & $\lambda_{-}$ & & & \\
\hline & & 0.1149 & 0.8988 & 3.0979 & 73.6520 & 82.4577 & & & $0.0092(0.9831)$ \\
\hline & MTS & $\mu$ & $\alpha$ & $C$ & $\lambda_{+}$ & $\lambda_{-}$ & & & \\
\hline & & 0.1150 & 0.8902 & 2.6734 & 104.5000 & 93.7954 & & & $0.0092(0.9837)$ \\
\hline January 11,2001 & KR & $\mu$ & $\alpha$ & $k_{+}$ & $k_{-}$ & $r_{+}$ & $r$ & $p_{+}=p_{-}$ & \\
\hline -January 11, 2006 & & 0.1229 & 0.9479 & 3408.7974 & 3087.4882 & 0.0134 & 0.0152 & & $0.0088(0.9897)$ \\
\hline & CGMY & $\mu$ & $\alpha$ & C & $\lambda_{+}$ & $\lambda_{-}$ & & & \\
\hline & MTS & $\begin{array}{c}0.1229 \\
\mu\end{array}$ & $\begin{array}{c}0.8935 \\
\alpha\end{array}$ & $\begin{array}{c}3.1667 \\
C\end{array}$ & $\begin{array}{c}73.7954 \\
\lambda_{+}\end{array}$ & $\begin{array}{c}82.4308 \\
\lambda_{-}\end{array}$ & & & $0.0088(0.9899)$ \\
\hline & & 0.1229 & 0.8878 & 2.6874 & 104.4998 & 93.6635 & & & $0.0088(0.9902)$ \\
\hline February 8, 2001 & $\mathrm{KR}$ & $\mu$ & $\alpha$ & $k_{+}$ & $k_{-}$ & $r_{+}$ & $r_{-}$ & $p_{+}=p_{-}$ & \\
\hline -February 8, 2006 & $C$ & 0.1063 & 0.8833 & 3497.4976 & 3201.4204 & 0.0126 & 0.0140 & 21.0621 & $0.0085(0.9936)$ \\
\hline & CGMY & $\mu$ & $\alpha$ & $\begin{array}{c}C \\
3-4508\end{array}$ & $\lambda_{+}+$ & $\lambda_{-}$ & & & $0 \Omega \Omega$ \\
\hline & MTS & $\begin{array}{c}0.1063 \\
\mu\end{array}$ & $\begin{array}{c}0.8785 \\
\alpha\end{array}$ & $\begin{array}{c}3.4508 \\
C\end{array}$ & $\begin{array}{c}75.1052 \\
\lambda_{+}\end{array}$ & $\begin{array}{c}83.0600 \\
\lambda\end{array}$ & & & $7(0.9908)$ \\
\hline & & 0.1063 & 0.8864 & 2.7082 & 104.0000 & 94.2105 & & & $0.0089(0.9890)$ \\
\hline March 15,2001 & KR & & & $k_{+}$ & $k_{-}$ & & $r$ & $p_{+}=p_{-}$ & \\
\hline -March 15, 2006 & $G G)$ & 0.1132 & 0.8207 & 3674.4888 & 3382.1180 & 0.0119 & 0.0132 & 19.2860 & $0.0086(0.9920)$ \\
\hline & CGMY & $\begin{array}{c}\mu \\
0.1132\end{array}$ & $\begin{array}{c}\alpha \\
0.8480\end{array}$ & $\begin{array}{c}C \\
4.0765\end{array}$ & $\begin{array}{c}\lambda_{+} \\
77.0189\end{array}$ & $\begin{array}{c}\lambda_{-} \\
84.9301\end{array}$ & & & $0.0090(0.9865)$ \\
\hline & MTS & $\mu$ & $\alpha$ & C & $\lambda_{+}$ & $\lambda_{-}$ & & & \\
\hline & & 0.1132 & 0.8672 & 2.9808 & 104.0000 & 94.3696 & & & $0.0091(0.9844)$ \\
\hline
\end{tabular}


Table 2: Risk-Neutral Parameters for the S\&P 500 Index Option

\begin{tabular}{|c|c|c|c|c|c|c|c|c|}
\hline Date & Model & & Parame & & & $\mathrm{AAE}$ & $\mathrm{APE}$ & RMSE \\
\hline June 8,2005 & $\begin{array}{c}\text { KR } \\
\text { CGMY } \\
\text { MTS }\end{array}$ & $\begin{array}{c}k_{+} \\
12542.3696 \\
\tilde{\lambda}_{+} \\
291.0561 \\
\tilde{\lambda}_{+} \\
345.3434\end{array}$ & $\begin{array}{c}k_{-} \\
66.8563 \\
\tilde{\lambda}_{+} \\
245.6197 \\
\tilde{\lambda}_{-} \\
289.0754\end{array}$ & $\begin{array}{c}\tilde{r}_{+} \\
0.0022\end{array}$ & $\begin{array}{c}\tilde{r}_{-} \\
0.0159\end{array}$ & $\begin{array}{l}0.0657 \\
0.0791 \\
0.0134\end{array}$ & $\begin{array}{l}0.9108 \\
1.0961 \\
1.3793\end{array}$ & $\begin{array}{l}1.0839 \\
1.2793 \\
1.5620\end{array}$ \\
\hline July 13,2005 & $\begin{array}{c}\text { KR } \\
\text { CGMY } \\
\text { MTS }\end{array}$ & $\begin{array}{c}\tilde{k}_{+} \\
5386.5504 \\
\tilde{\lambda}_{+} \\
356.3451 \\
\tilde{\lambda}_{+} \\
430.1860\end{array}$ & $\begin{array}{c}\tilde{k}_{\overline{1}} \\
85.1748 \\
\tilde{\lambda}_{+} \\
297.7620 \\
\tilde{\lambda}_{-} \\
355.6672\end{array}$ & $\begin{array}{c}\tilde{r}_{+} \\
0.0020\end{array}$ & $\begin{array}{c}\tilde{r}_{-} \\
0.0127\end{array}$ & $\begin{array}{l}0.0401 \\
0.0471 \\
0.0104\end{array}$ & $\begin{array}{l}0.8865 \\
1.0409 \\
1.0964\end{array}$ & $\begin{array}{l}1.0797 \\
1.2455 \\
1.2878\end{array}$ \\
\hline August 10,2005 & $\begin{array}{c}\text { KR } \\
\text { CGMY } \\
\text { MTS }\end{array}$ & $\begin{array}{c}\tilde{k}_{+} \\
13532.0787 \\
\tilde{\lambda}_{+} \\
281.3249 \\
\tilde{\lambda}_{+} \\
318.7796 \\
\end{array}$ & $\begin{array}{c}\tilde{k}_{-} \\
71.4528 \\
\tilde{\lambda}_{+} \\
235.9410 \\
\tilde{\lambda}_{-} \\
266.9580\end{array}$ & $\begin{array}{c}\tilde{r}_{+} \\
0.0023\end{array}$ & $\begin{array}{c}\tilde{r}_{-} \\
0.0158\end{array}$ & $\begin{array}{l}0.0238 \\
0.0266 \\
0.0154\end{array}$ & $\begin{array}{l}1.2231 \\
1.3662 \\
2.0858\end{array}$ & $\begin{array}{r}1.5566 \\
1.7245 \\
2.3224 \\
\end{array}$ \\
\hline September 14,2005 & $\begin{array}{c}\text { KR } \\
\text { CGMY } \\
\text { MTS }\end{array}$ & $\begin{array}{c}\dot{k}_{+} \\
21807.5069 \\
\tilde{\lambda}_{+} \\
260.8052 \\
\tilde{\lambda}_{+} \\
317.4894 \\
\end{array}$ & $\begin{array}{c}\bar{k}_{-} \\
45.8005 \\
\tilde{\lambda}_{+} \\
220.0867 \\
\tilde{\lambda}_{-} \\
265.8617\end{array}$ & $\begin{array}{c}\tilde{r}_{+} \\
0.0023\end{array}$ & $\begin{array}{c}\tilde{r}_{-} \\
0.0195\end{array}$ & $\begin{array}{l}0.0502 \\
0.0562 \\
0.0206\end{array}$ & $\begin{array}{l}1.6336 \\
1.8315 \\
2.0149\end{array}$ & $\begin{array}{l}1.8841 \\
2.1030 \\
2.2407\end{array}$ \\
\hline October 12,2005 & $\begin{array}{c}\text { KR } \\
\text { CGMY } \\
\text { MTS }\end{array}$ & $\begin{array}{c}\tilde{k}_{+} \\
13646.4725 \\
\tilde{\lambda}_{+} \\
227.6580 \\
\tilde{\lambda}_{+} \\
281.8887\end{array}$ & $\begin{array}{c}\tilde{k}_{-} \\
52.7056 \\
\tilde{\lambda}_{+} \\
194.4653 \\
\tilde{\lambda}_{-} \\
239.4128\end{array}$ & $\begin{array}{c}\tilde{r}_{+} \\
0.0028\end{array}$ & $\begin{array}{c}\tilde{r}_{-} \\
0.0200\end{array}$ & $\begin{array}{l}0.0358 \\
0.0500 \\
0.0425\end{array}$ & $\begin{array}{l}0.4529 \\
0.6319 \\
0.5996\end{array}$ & $\begin{array}{l}0.5363 \\
0.7516 \\
0.7466\end{array}$ \\
\hline November 9, 2005 & $\begin{array}{c}\text { KR } \\
\text { CGMY } \\
\text { MTS }\end{array}$ & $\begin{array}{c}\tilde{k}_{+} \\
8653.7220 \\
\tilde{\lambda}_{+} \\
285.8952 \\
\tilde{\lambda}_{+} \\
329.3268\end{array}$ & $\begin{array}{c}\tilde{k}_{-} \\
64.5551 \\
\tilde{\lambda}_{+} \\
243.6277 \\
\tilde{\lambda}_{-} \\
279.6664\end{array}$ & $\begin{array}{c}\tilde{r}_{+} \\
0.0023\end{array}$ & $\begin{array}{c}\tilde{r}_{-} \\
0.0160\end{array}$ & $\begin{array}{l}0.0307 \\
0.0351 \\
0.0086\end{array}$ & $\begin{array}{l}1.1898 \\
1.3584\end{array}$ & $\begin{array}{l}1.3543 \\
1.5441 \\
1.4285\end{array}$ \\
\hline December 14,2005 & $\begin{array}{c}\text { KR } \\
\text { CGMY } \\
\text { MTS }\end{array}$ & $\begin{array}{c}k_{+} \\
8831.8398 \\
\tilde{\lambda}_{+} \\
307.0166 \\
\tilde{\lambda}_{+} \\
373.4433\end{array}$ & $\begin{array}{c}\dot{k}_{-} \\
79.9054 \\
\tilde{\lambda}_{+} \\
258.8013 \\
\tilde{\lambda}_{-} \\
313.9098\end{array}$ & $\begin{array}{c}\tilde{r}_{+} \\
0.0022\end{array}$ & $\begin{array}{c}\tilde{r}_{-} \\
0.0141\end{array}$ & $\begin{array}{l}0.0460 \\
0.0520 \\
0.0148\end{array}$ & $\begin{array}{l}1.4435 \\
1.6309 \\
1.7318\end{array}$ & $\begin{array}{l}1.6268 \\
1.8310 \\
1.8733\end{array}$ \\
\hline January 11,2006 & $\begin{array}{c}\text { KR } \\
\text { CGMY } \\
\text { MTS }\end{array}$ & $\begin{array}{c}\tilde{k}_{+} \\
13726.6419 \\
\tilde{\lambda}_{+} \\
358.5781 \\
\tilde{\lambda}_{+} \\
433.7411\end{array}$ & $\begin{array}{c}\tilde{k}_{-} \\
69.2806 \\
\tilde{\lambda}_{+} \\
300.7159 \\
\tilde{\lambda}_{-} \\
361.1325\end{array}$ & $\begin{array}{c}\tilde{r}_{+} \\
0.0017\end{array}$ & $\begin{array}{c}\tilde{r}_{-} \\
0.0132\end{array}$ & $\begin{array}{l}0.0226 \\
0.0275 \\
0.0201\end{array}$ & $\begin{array}{l}0.7375 \\
0.8973 \\
0.8927\end{array}$ & $\begin{array}{l}0.8317 \\
1.0106 \\
1.0116\end{array}$ \\
\hline February 8, 2006 & $\begin{array}{c}\text { KR } \\
\text { CGMY } \\
\text { MTS }\end{array}$ & $\begin{array}{c}\tilde{k}_{+} \\
12043.3247 \\
\tilde{\lambda}_{+} \\
282.3814 \\
\tilde{\lambda}_{+} \\
343.8596\end{array}$ & $\begin{array}{c}\tilde{k}_{-} \\
65.8378 \\
\tilde{\lambda}_{+} \\
242.4361 \\
\tilde{\lambda}_{-} \\
294.4044\end{array}$ & $\begin{array}{c}\tilde{r}_{+} \\
0.0023\end{array}$ & $\begin{array}{c}\tilde{r}_{-} \\
0.0158\end{array}$ & $\begin{array}{l}0.0313 \\
0.0400 \\
0.0260\end{array}$ & $\begin{array}{l}0.6890 \\
0.8805 \\
0.9025\end{array}$ & $\begin{array}{l}0.7942 \\
1.0129 \\
1.0364\end{array}$ \\
\hline March 15, 2006 & $\begin{array}{c}\text { KR } \\
\text { CGMY } \\
\text { MTS }\end{array}$ & $\begin{array}{c}\dot{k}_{+} \\
24676.3763 \\
\tilde{\lambda}_{+} \\
293.4144 \\
\tilde{\lambda}_{+} \\
356.0416\end{array}$ & $\begin{array}{c}\bar{k}_{-} \\
77.8299 \\
\tilde{\lambda}_{+} \\
250.4869 \\
\tilde{\lambda}_{-} \\
302.9546\end{array}$ & $\begin{array}{c}\tilde{r}_{+} \\
0.0022\end{array}$ & $\begin{array}{c}\tilde{r}_{-} \\
0.0145\end{array}$ & $\begin{array}{l}0.0405 \\
0.0459 \\
0.0158\end{array}$ & $\begin{array}{l}1.4528 \\
1.6464 \\
1.7091\end{array}$ & $\begin{array}{l}1.6475 \\
1.8519 \\
1.8766\end{array}$ \\
\hline
\end{tabular}

Table 3: Estimated normal-GARCH parameters

\begin{tabular}{ccccc}
\hline & $\beta_{1}$ & $\alpha_{1}$ & $\alpha_{0}$ & $\lambda$ \\
\hline SPX & 0.8489 & 0.1423 & $1.537282 \mathrm{E}-5$ & 0.0471 \\
INTC & 0.8753 & 0.1166 & $8.874328 \mathrm{E}-5$ & 0.0431 \\
MSFT & 0.8664 & 0.1275 & $4.921977 \mathrm{E}-5$ & 0.0390 \\
AMZN & 0.9879 & 0.0111 & $9.946677 \mathrm{E}-6$ & 0.0436 \\
\hline
\end{tabular}


Table 4: Estimated parameters of the innovation processes for the tempered stable GARCH models

\begin{tabular}{lllllll}
\hline Ticker & Model & \multicolumn{5}{c}{ Parameters } \\
\hline SPX & stdCTS & $\alpha=1.7309$ & $\lambda_{+}=0.0343$ & $\lambda_{-}=0.0340$ & & \\
& stdMTS & $\alpha=1.7904$ & $\lambda_{+}=0.0343$ & $\lambda_{-}=0.0353$ & & \\
& stdKR & $\alpha=1.7591$ & $r_{+}=29.1424$ & $r_{-}=69.5218$ & $p_{+}=12.6231$ & $p_{-}=7.7217$ \\
\hline INTC & stdCTS & $\alpha=1.5695$ & $\lambda_{+}=0.0975$ & $\lambda_{-}=0.0965$ & & \\
& stdMTS & $\alpha=1.6748$ & $\lambda_{+}=0.0975$ & $\lambda_{-}=0.0975$ & & \\
& stdKR & $\alpha=1.6633$ & $r_{+}=10.2595$ & $r_{-}=58.6107$ & $p_{+}=9.0927$ & $p_{-}=4.8600$ \\
\hline MSFT & stdCTS & $\alpha=1.5930$ & $\lambda_{+}=0.0756$ & $\lambda_{-}=0.0749$ & & \\
& stdMTS & $\alpha=1.6899$ & $\lambda_{+}=0.0756$ & $\lambda_{-}=0.0758$ & & \\
& stdKR & $\alpha=1.5971$ & $r_{+}=9.3119$ & $r_{-}=53.6227$ & $p_{+}=-0.2670$ & $p_{-}=13.9722$ \\
\hline AMZN & stdCTS & $\alpha=1.4899$ & $\lambda_{+}=0.0737$ & $\lambda_{-}=0.0975$ & & \\
& stdMTS & $\alpha=1.4993$ & $\lambda_{+}=0.2283$ & $\lambda_{-}=0.0743$ & & \\
& stdKR & $\alpha=1.3833$ & $r_{+}=6.0613$ & $r_{-}=15.7081$ & $p_{+}=1.7226$ & $p_{-}=7.0348$ \\
\hline
\end{tabular}

Table 5: Statistic of the goodness of fit tests

\begin{tabular}{llccc}
\hline Ticker & Model & KS & $p$-value & AD \\
\hline SPX & normal-GARCH & 0.0979 & 0.0000 & 1.7958 \\
& CTS-GARCH & 0.0257 & 0.0723 & 0.1317 \\
& MTS-GARCH & 0.0260 & 0.0677 & 0.1459 \\
& KR-GARCH & $\mathbf{0 . 0 2 4 2}$ & $\mathbf{0 . 1 0 6 6}$ & 0.1437 \\
\hline INTC & normal-GARCH & 0.1032 & 0.0000 & 7.9422 \\
& CTS-GARCH & 0.0226 & 0.1530 & 0.1482 \\
& MTS-GARCH & 0.0226 & 0.1543 & 0.1564 \\
& KR-GARCH & $\mathbf{0 . 0 1 7 7}$ & $\mathbf{0 . 4 1 2 9}$ & 0.1769 \\
\hline MSFT & normal-GARCH & 0.1053 & 0.0000 & 0.5452 \\
& CTS-GARCH & 0.0288 & 0.0310 & 0.0966 \\
& MTS-GARCH & 0.0286 & 0.0332 & 0.1057 \\
& KR-GARCH & $\mathbf{0 . 0 1 5 1}$ & $\mathbf{0 . 6 1 8 6}$ & 0.1157 \\
\hline AMZN & normal-GARCH & 0.1208 & 0.0000 & $3.044068 \mathrm{E}+20$ \\
& CTS-GARCH & 0.0156 & 0.9209 & 0.9891 \\
& MTS-GARCH & 0.0427 & 0.0212 & 0.6849 \\
& KR-GARCH & $\mathbf{0 . 0 1 4 9}$ & $\mathbf{0 . 9 4 3 8}$ & 0.7856 \\
\hline
\end{tabular}

Table 6: Errors between the market and model prices of options

\begin{tabular}{lccc}
\hline & APE & AAE & RMSE \\
\hline BS & 0.1850 & 3.4441 & 4.3050 \\
CGMY & 0.1850 & 3.4296 & 4.2380 \\
Normal-GARCH & 0.1387 & 2.5709 & 3.2167 \\
CTS-GARCH & 0.0722 & 1.3391 & 1.6307 \\
MTS-GARCH & $\mathbf{0 . 0 4 5 7}$ & $\mathbf{0 . 8 4 6 3}$ & $\mathbf{1 . 1 0 1 4}$ \\
KR-GARCH & 0.0816 & 1.5120 & 1.8084 \\
\hline
\end{tabular}




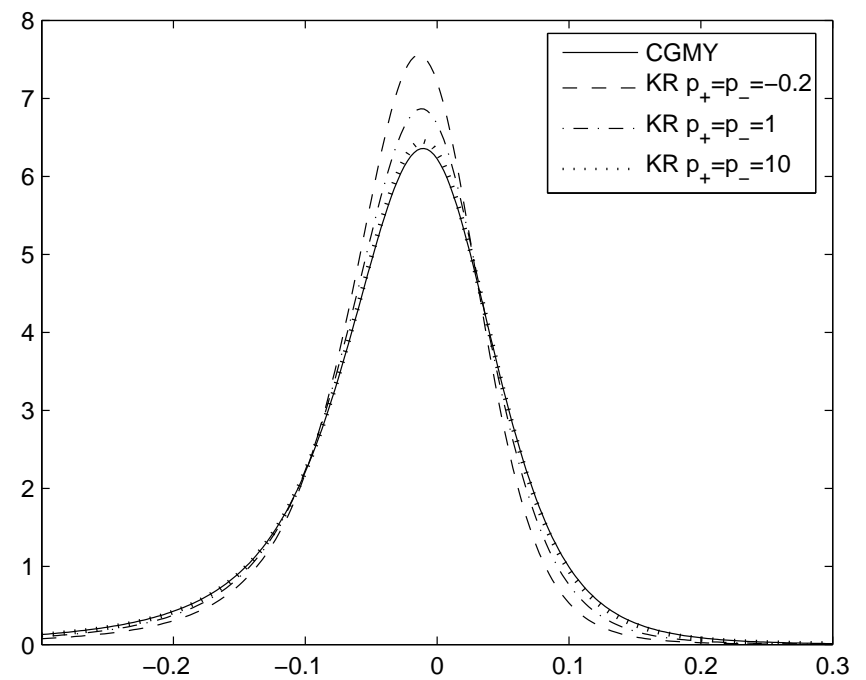

Figure 1: Probability density of the CGMY distribution with parameters $C=0.01, G=2, M=10$, $Y=1.25$, and the KR distributions with $\alpha=Y, k_{ \pm}=C(Y+p) r_{ \pm}^{-\alpha}, r_{+}=1 / M, r_{-}=1 / G$, where $p=p_{+}=p_{-} \in\{-0.25,1,10\}$.
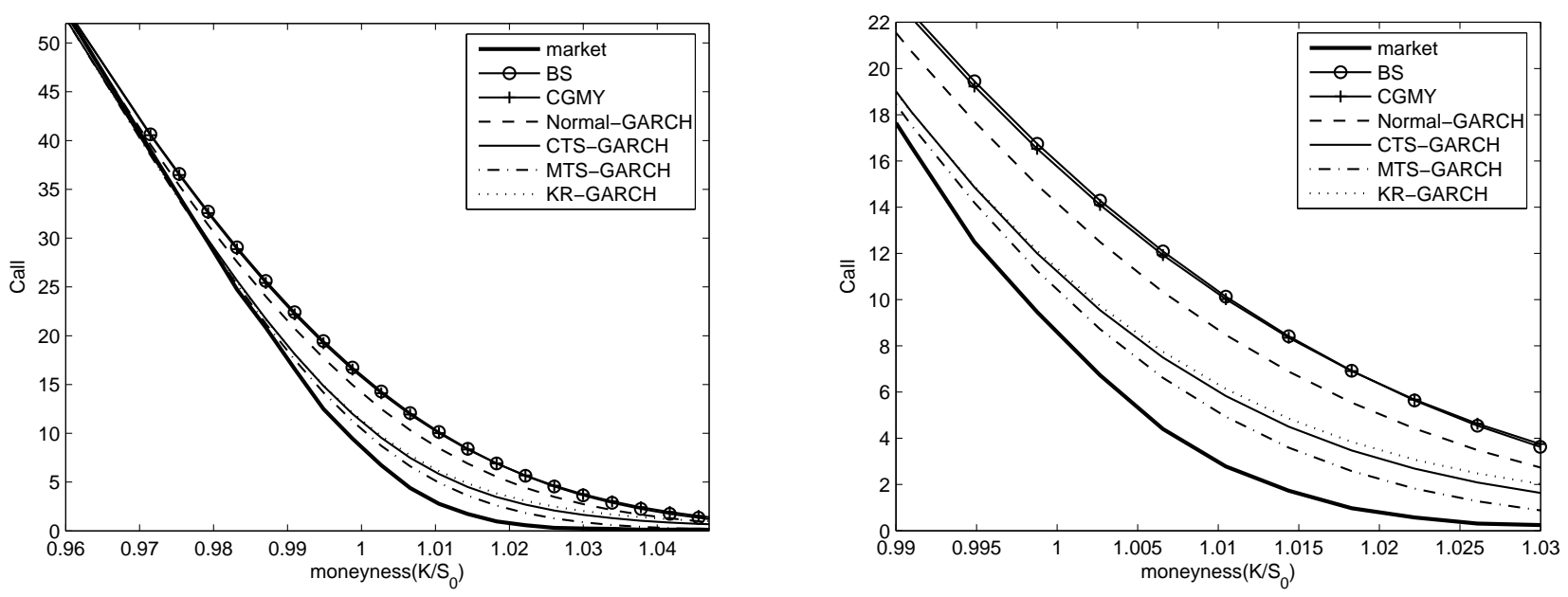

Figure 2: Call prices 


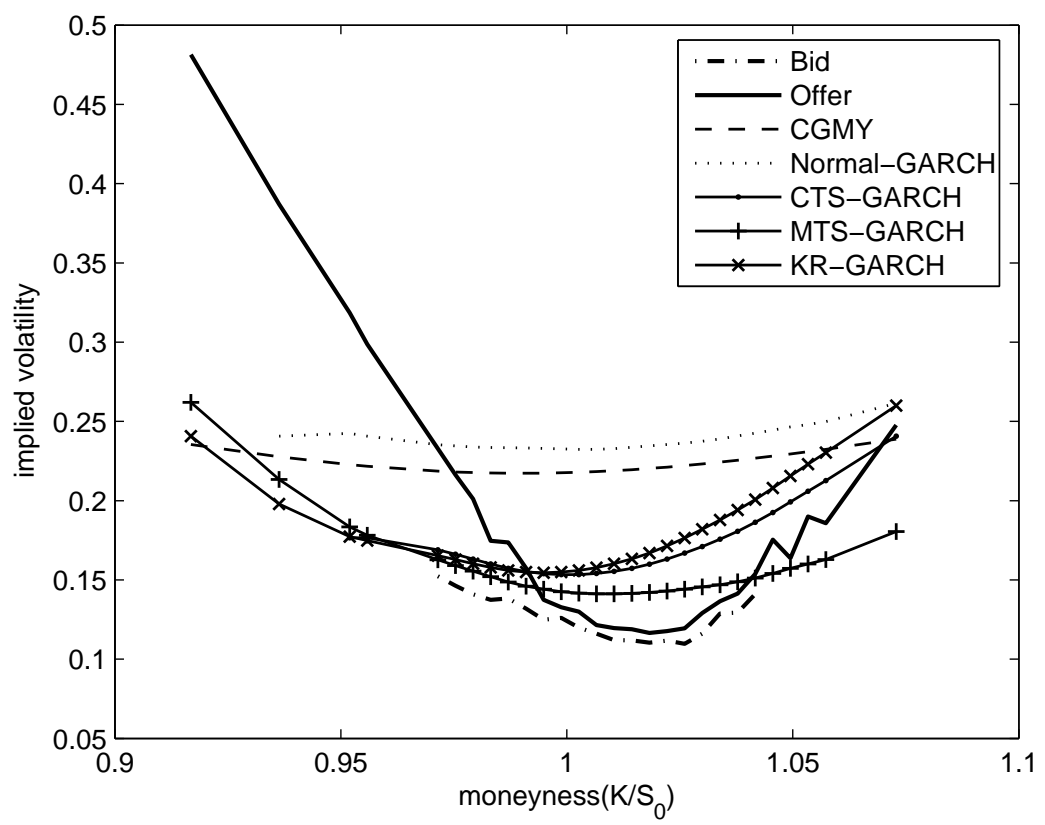

Figure 3: Implied volatility 